\title{
LIPSCHITZ SPACES ON STRATIFIED GROUPS
}

\author{
BY
}

STEVEN G. KRANTZ ${ }^{1}$

\begin{abstract}
Let $G$ be a connected, simply connected nilpotent Lie group. Call $G$ stratified if its Lie algebra $g$ has a direct sum decomposition $g=V_{1} \oplus \cdots \oplus V_{m}$ with $\left[V_{i}, V_{j}\right]=V_{i+j}$ for $i+j<m,\left[V_{i}, V_{j}\right]=0$ for $i+j>m$. Let $\left\{X_{1}, \ldots, X_{n}\right\}$ be a vector space basis for $V_{1}$. Let $f \in C(G)$ satisfy $\left\|f\left(g \exp X_{i} \cdot\right)\right\| \in \Lambda_{\alpha}(R)$, uniformly in $g \in G$, where $\Lambda_{\alpha}$ is the usual Lipschitz space and $0<\alpha<\infty$. It is proved that, under these circumstances, it holds that $f \in \Gamma_{\alpha}(G)$ where $\Gamma_{\alpha}$ is the nonisotropic Lipschitz space of Folland. Applications of this result to interpolation theory, hypoelliptic partial differential equations, and function theory are provided.
\end{abstract}

0. Let $G$ be a finite-dimensional, connected, simply-connected Lie group and $\mathrm{g}$ its Lie algebra. If $X, Y \in \mathfrak{g}$, then their Lie bracket $[X, Y]=X Y-Y X \in \mathfrak{g}$ will be called a first order commutator. If $Z \in \mathfrak{g}$ is an $m$ th order commutator and $W \in \mathfrak{g}$ then $[Z, W]$ is an $(m+1)$ st order commutator. If there is an $m>0, m \in \mathbf{Z}$, so that all $m$ th order commutators in $g$ vanish, then we say that $g$ (and hence $G$ ) is nilpotent.

A nilpotent Lie algebra (and its associated Lie group $G$ ) is stratified if there is a direct sum vector space decomposition

$$
\mathrm{g}=V_{1} \oplus \cdots \oplus V_{m}
$$

so that each element of $V_{j}, 2 \leqslant j \leqslant m$, is a linear combination of $(j-1)$ th order commutators of elements of $V_{1}$. Equivalently, (1.1) is a stratification provided $\left[V_{i}, V_{j}\right]=V_{i+j}$ whenever $i+j \leqslant m$ and $\left[V_{i}, V_{j}\right]=0$ otherwise.

Stratified nilpotent Lie groups are equipped with a natural dilation structure and are therefore a setting for the study of subelliptic partial differential equations [8], [6], [17]. The purpose of the present work is to study some function spaces which arise in this context. The results are close in spirit to the subelliptic estimates which hold in the Lipschitz category for certain invariant differential operators on stratified groups (see [6]). The methods presented here are of some interest because (i) they are obtained by a method of implicit estimation not common to the study of Lipschitz functions, (ii) they make a somewhat novel use of the calculus of finite differences, (iii) they serve to clarify the role which homogeneity exerts over the estimates, (iv) they serve to explain (see §11) why the Hörmander sum-of-squares operator is subelliptic. The results themselves are also of interest because they may

Received by the editors July 9, 1978 and, in revised form, September 17, 1980.

1980 Mathematics Subject Classification. Primary 22E25, 35H05; Secondary 41A05, 26B05, 26B35.

${ }^{1}$ Research supported in part by NSF Grant MCS 77-02213. The author is grateful to the Université de Paris-Sud for its hospitality during a portion of this work.

(C) 1982 American Mathematical Society 0002-9947/82/0000-0308/\$08.00 
be used to reduce certain results (such as theorems about interpolation of linear operators) to one-dimensional theorems.

Recall the existence of the exponential map $\exp _{e}: \mathfrak{g} \rightarrow G$. It provides, on a connected, simply-connected nilpotent Lie group, a globally defined diffeomorphism of $\mathfrak{g}$ onto $G$. In particular, the underlying topological space of $G$ is $\mathbf{R}^{N}$, some $N$. This fact will play no essential role in what follows, because the proofs are local in nature. It will, however, serve to simplify the statements and proofs of some results.

In what follows, we use the classical Lipschitz spaces as defined and studied in [18]. On open subsets $U \subseteq \mathbf{R}^{N}$, they are denoted by $\Lambda_{\alpha}(U), 0<\alpha<\infty$. Let $\mathscr{B} \mathcal{C}(U)$ be the bounded continuous functions under sup norm. We have

$$
\begin{aligned}
& \Lambda_{\alpha}(U)=\left\{f \in \mathscr{B} C: \sup _{x, x+h \in U}|f(x+h)-f(x)| /|h|^{\alpha}\right. \\
& \left.+\|f\|_{L^{\infty}} \equiv\|f\|_{\Lambda_{\alpha}(U)}<\infty\right\}, \quad 0<\alpha<1 ; \\
& \Lambda_{1}(U)=\left\{f \in \mathscr{B} \mathcal{C}(U): \sup _{x, x+h, x-h \in U}|f(x+h)+f(x-h)-2 f(x)| /|h|\right. \\
& \left.+\|f\|_{L^{\infty}} \equiv\|f\|_{\Lambda_{1}(U)}<\infty\right\} \\
& \Lambda_{\alpha}(U)=\left\{f \in \mathscr{B} \mathcal{C}(U): \sum_{j=1}^{N}\left\|\frac{\partial f}{\partial x_{j}}\right\|_{\Lambda_{\alpha-1}(U)}+\|f\|_{L^{\infty}(U)} \equiv\|f\|_{\Lambda_{\alpha}(U)}<\infty\right\}, \\
& \alpha>1 .
\end{aligned}
$$

MAIN THEOREM. Suppose that $G$ is a (connected, simply-connected) nilpotent Lie group and suppose that its Lie algebra $g$ has the stratification $g=V_{1} \oplus \cdots \oplus V_{m}$. Let $\left\{X_{1}, \ldots, X_{n}\right\}$ be a vector space basis for $V_{1}$ over $\mathbf{R}$. Let $f: G \rightarrow \mathbf{C}$ be an everywhere-defined function. Let $\alpha>0$.

Suppose there is a $C_{0}>0$ so that for every $g \in G$, every $j \in\{1, \ldots, n\}$, the functions

$$
f_{g, j}: \mathbf{R} \rightarrow \mathbf{C}, \quad f_{g j}(t)=f\left(g \exp X_{j} t\right)
$$

satisfy

$$
\left\|f_{g_{j}}\right\|_{\Lambda_{a}(\mathbf{R})} \leqslant C_{0}
$$

Then

(i) $f \in \Lambda_{\alpha / m}^{\text {loc }}$ in any local $C^{\infty}$ coordinates frame on $G$;

(ii) if $Y_{i} \stackrel{\alpha / m}{\in} V_{j(i)}, i=1, \ldots, k$, and if $\gamma=1-\sum_{i=1}^{k} j(i) / \alpha>0$ then $\left(Y_{1} \cdots Y_{k}\right) f$ exists and is continuous;

(iii) there is a $C>0$ so that (with notation as in (ii)) with $Z \in V_{l}$, any $1 \leqslant l \leqslant m$, and $g \in G$, it holds that

$$
\left\|\left(Y_{1} \cdots Y_{k}\right) f(g \exp Z \cdot)\right\|_{\Lambda_{\alpha \gamma / l}}<C \cdot C_{0} .
$$

These estimates cannot be improved. 
The plan of the paper is as follows. In $\$ 1$ we recall some notions from the theory of stratified nilpotent groups (as developed in [6]) and recast the Main Theorem in terms of Folland's terminology. In $\$ 2$ we develop some fairly standard facts about Lipschitz spaces which will be needed later on.

Next we turn to the proof of the Main Theorem. The case $\alpha \in \mathbf{Z}$ must be handled separately. We establish an a priori inequality for $f \in \mathscr{B} \bigodot \cap C^{\infty}, \alpha \notin \mathbf{Z}$, in $\$ \S 3-5 . \$ \$ 6$ and 7 show how to pass from the $a$ priori inequality to the full result, $\alpha \notin \mathbf{Z}$. $\$ 8$ shows how to handle the case $\alpha \in \mathbf{Z}$. \$\$9-12 contain variants of the Main Theorem and applications to interpolation theory, function theory, and partial differential equations.

I would like to thank the referee for many constructive suggestions which helped to improve the exposition in this paper, and for directing my attention to useful sources in the literature.

1. Some known results on stratified groups. In order to establish the meaning of the Main Theorem, we connect it with the spaces $\Gamma_{\alpha}$ which have been exploited in earlier work ([8], [17], [6], [7]).

Recall (see [6] for details) that a stratified nilpotent Lie algebra $g$ admits a family of dilations: this is a one-parameter family $\left\{\gamma_{r}: 0<r<\infty\right\}$ of automorphisms of $g$ of the form $\gamma_{r}=\exp (A \log r)$ where $A$ is a diagonalizable linear transformation of $\mathfrak{g}$ with positive eigenvalues. On a stratified Lie algebra it is convenient to use the dilations

$$
\gamma_{r}\left(Y_{1}+\cdots+Y_{m}\right)=r Y_{1}+\cdots+r^{m} Y_{m}, \quad Y_{j} \in V_{j}
$$

Since the exponential map exp: $\mathfrak{g} \rightarrow G$ is a global diffeomorphism, the $\left\{\gamma_{r}\right\}$ induce a one-parameter family of automorphisms of $G$ which we also denote by $\left\{\gamma_{r}\right\}$. The number $Q=\operatorname{trace} A=\sum_{j=1}^{m} j \operatorname{dim} V_{j}$ is called the homogeneous dimension of $\mathrm{g}$.

If $f: G \rightarrow \mathbf{C}$ we call $f$ homogeneous of degree $\lambda, \lambda \in \mathbf{C}$, if $f \circ \gamma_{r}=r^{\lambda} f$, all $r>0$. A homogeneous norm on $G$ is a function $x \rightarrow|x| \in \mathbf{R}^{+}$which is homogeneous of degree 1 and such that (i) $|x|=0$ iff $x=0=$ identity, (ii) $|x|=\left|x^{-1}\right|$ for all $x$. A convenient such norm on a stratified group, which is compatible with the dilations described above, is given as follows. Equip $g$ with some metric and associated norm \|\| so that the $V_{j}$ are mutually orthogonal. If $Y \in \mathfrak{g}, Y=\sum_{j=1}^{m} Y_{j}, Y_{j} \in V_{j}$, then $\left\|\gamma_{r} Y\right\|=\left(\sum r^{2 j}\left\|Y_{j}\right\|^{2}\right)^{1 / 2}$. Now if $g \in G$, define $|g|$ to be the unique $r>0$ so that $\left\|\gamma_{1 / r} \exp ^{-1} g\right\|=1$. Note that, with this definition, $|\exp Y|<C\|Y\|^{1 / m}$.

We fix once and for all the dilations and homogeneous norm described here. If $X_{1}, \ldots, X_{n}$ is the basis for $V_{1}$ described in the Main Theorem then we will assume that $\left\|X_{j}\right\|=1, j=1, \ldots, n$.

Now we recall the Lipschitz spaces of Folland and Stein [8], [6]: Let $\mathscr{B} \mathcal{C}$ denote the bounded continuous functions on $G$, under supremum norm.

If $0<\alpha<1$, let

$$
\Gamma_{\alpha}=\left\{f \in \mathscr{B} \bigodot:\|f\|_{\Gamma_{\alpha}} \equiv \sup _{\substack{x, y \in G \\ y \neq 0}}|f(x y)-f(x)| /|y|^{\alpha}+\|f\|_{L^{\infty}}<\infty\right\} .
$$


For $\alpha=1$, let

$$
\Gamma_{1}=\left\{f \in \mathscr{B} C:\|f\|_{\Gamma_{1}} \equiv \sup _{\substack{x, y \in G \\ y \neq 0}}\left|f(x y)+f\left(x y^{-1}\right)-2 f(x)\right| /|y|+\|f\|_{L^{\infty}}<\infty\right\} .
$$

If $\alpha=l+\alpha^{\prime}, l=1,2, \ldots, 0<\alpha^{\prime} \leqslant 1$, let

$$
\Gamma_{\alpha}=\left\{f \in \mathscr{B} \circlearrowright:\|f\|_{\Gamma_{\alpha}}=\sup _{\substack{Y_{i} \in V_{j(i)} \\ 1<i<k \\ \Sigma_{j} j(i)<l}}\left\|Y_{1} \cdots Y_{k} f\right\|_{\Gamma_{\alpha^{\prime}}}+\|f\|_{L^{\infty}}<\infty\right\} .
$$

The following lemma is a slight modification of Lemma 5.1 of [6]; similar results may be found in [11].

LEMMA 1.1. Let $X_{1}, \ldots, X_{n}$ be a fixed basis for $V_{1}$. There is a constant $C>0$ and an integer $N>0$ so that every $g \in G$ may be written in the form $g=x_{1} \cdots x_{N}$, $x_{j}=\exp s_{j} X_{i},\left|x_{j}\right| \leqslant C|x|$.

Proof. Following Folland [6], we define a family of maps from

$$
B=\left\{Y \in V_{1}:|\exp Y| \leqslant 1\right\}
$$

into $G$. If $Y \in B, Y=\Sigma t_{i} X_{i}$, let

$$
\varphi^{0}(Y)=\exp t_{1} X_{1} \cdots \exp t_{n} X_{n} .
$$

If $1 \leqslant p \leqslant m-1,1 \leqslant i_{1}, \ldots, i_{p} \leqslant n$, let

$$
\varphi_{i_{1}}^{p} \cdots_{p}(Y)=\left[\exp X_{i_{p}},\left[\cdots,\left[\exp X_{i_{2}},\left[\exp X_{i_{1}}, \varphi^{0}(Y)\right]\right] \cdots\right]\right] .
$$

The Campbell-Hausdorff formula implies that the differential $D_{0} \varphi_{i_{1}}^{p} \cdots_{j}$ of $\varphi_{i_{1}}^{p} \cdots_{p}$ at 0 is a map from $V_{1}$ to $g$ given by

$$
D_{0} \varphi^{0}(Y)=Y, \quad D_{0} \varphi_{i_{1}}^{p} \ldots i_{p}(Y)=\left[X_{i_{p}},\left[\cdots,\left[X_{i_{2}},\left[X_{i_{1}}, Y\right]\right] \cdots\right]\right] .
$$

Now define

$$
\varphi: \frac{B \times \cdots \times B}{\left(\sum_{p=0}^{m-1} n^{p}\right) \text { times }} \rightarrow G
$$

by

$$
\varphi(Y)=\prod_{p=0}^{m-1} \prod_{\substack{1<i_{i}<n \\ 1<l<p}} \varphi_{i_{1} \cdots j_{p}}^{p}(Y) .
$$

Once again the Campbell-Hausdorff formula implies that $D_{0} \varphi$, being the sum of the differentials of the $\varphi^{p}$ 's, is surjective onto $g$ (we use here the definition of the stratification). The implicit function theorem now yields that $\varphi$ is surjective onto a neighborhood $U=\left\{x:|x|<r_{0}\right\}$ of $0 \in G$. Thus every element of $U$ is at most a $\sum_{p=0}^{m-1} n^{p}$ order commutator of elements $\exp a_{l} X_{i}$, hence a product of at most $N=\sum_{p=0}^{m-1} n^{p}\left(3 \cdot 2^{p}-2\right)$ elements $\exp a_{l} X_{i_{l}}$. Each of the elements $\exp a_{l} X_{i_{l}}$ has norm not greater than 1 . By dilation, the result now follows with $C=1 / r_{0}$.

Now let $\alpha>0$ and let $f \in \Gamma_{\alpha}(G)$. It follows that

$$
\sup _{g \in G}\left\|f\left(g \exp X_{i} \cdot\right)\right\|_{\Lambda_{\alpha}}<\infty, \quad i=1, \ldots, n \text {. }
$$


So $f$ satisfies the hypotheses, hence the conclusions, of the Main Theorem (that it satisfies the conclusion may also be verified directly).

Conversely, suppose $f$ satisfies the conclusion of the Main Theorem. First let $0<\alpha<1$. Let $g \in G, h \in G$. According to Lemma 1.1, write $h=h_{1} \cdots h_{N}$, $h_{j}=\exp s_{j} X_{i_{j}}$. Then

$$
\begin{aligned}
|f(g h)-f(g)| & \leqslant \sum_{j=1}^{N}\left|f\left(g h_{1} \cdots h_{j}\right)-f\left(g h_{1} \cdots h_{j-1}\right)\right| \\
& \leqslant C \cdot C_{0} \sum\left|s_{j}\right|^{\alpha}<C|h|^{\alpha} .
\end{aligned}
$$

So $f \in \Gamma_{\alpha}$. If $k<\alpha<k+1,1 \leqslant k \in \mathbf{Z}$, then for any $X_{i_{1}}, \ldots, X_{i_{k}}$ we have that $X_{i_{1}} \cdots X_{i_{k}} f$ satisfies the hypotheses, hence the conclusions, of the Main Theorem with respect to $\alpha^{\prime}=\alpha-k$. So $X_{i_{1}} \cdots X_{i_{k}} f \in \Gamma_{\alpha^{\prime}}$ and $f \in \Gamma_{\alpha}$.

The case $\alpha \in \mathbf{Z}$ will be handled by a sort of interpolation argument in $\S 9$. Taking this for granted for now, we may restate the conclusion of the Main Theorem quite simply as $f \in \Gamma_{\alpha}$.

As a biproduct of the preceding argument we have proved

LEMMA 1.2. The Main Theorem holds for $\alpha<1$. In particular, for $\alpha<1, f \in$ $\Lambda_{\alpha / m}^{\operatorname{loc}}\left(\mathbf{R}^{N}\right)$ when $G$ is equipped with the Lie algebra coordinates coming from the exponential map.

Proof. For the first assertion, simply refer to the argument above using Lemma 1.1. For the second, see [6, p. 5.17ff].

2. Some classical results about Lipschitz spaces. We collect here some results of a fairly classical nature about Lipschitz spaces on Euclidean space. Where we do not provide proofs, we give standard references.

LeMma 2.1. Let $0 \leqslant k<\alpha<k+1 \in \mathbf{Z}$. If $f \in \Lambda_{\alpha}(\mathbf{R})$, then

$$
f(x+h)=f(x)+h \cdot f^{\prime}(x)+\cdots+\left(h^{k} / k !\right) f^{(k)}(x)+O\left(|h|^{\alpha}\right),
$$

all $x, h \in \mathbf{R}$.

Proof. The result for $0<\alpha<1$ is trivial. Inductively obtain the result for $\alpha>1$ by applying the result for $\alpha-1$ to $f^{\prime}$.

If $f: \mathbf{R} \rightarrow \mathbf{C}, x, h \in \mathbf{R}, k=1,2,3, \ldots$, let

$$
\Delta_{h}^{k} f(x)=\left.\Delta_{h}^{k} f(\cdot)\right|_{x}=\sum_{l=0}^{k}(-1)^{l+k}\left(\begin{array}{c}
k \\
l
\end{array}\right) f(x+(2 l-k) h) .
$$

Let $\Delta_{h}^{0} f(x)=f(x)$. Finite differences may be used to characterize $\Lambda_{\alpha}$ as follows:

LEMMA 2.2. Let $0<\alpha<k \in \mathbf{Z}$. Let $f: \mathbf{R} \rightarrow \mathbf{C}$ be continuous. Then $f \in \Lambda_{\alpha}(\mathbf{R})$ if and only if

$$
\sup _{\substack{x, h \\ h \neq 0}}\left|\Delta_{h}^{k} f(x)\right| /|h|^{\alpha}+\|f\|_{L^{\infty}}=C_{0}<\infty .
$$

The numbers $C_{0}$ and $\|f\|_{\Lambda_{\alpha}}$ are comparable. 
Proof. See [4].

Lemma 2.3. If $f, g: \mathbf{R} \rightarrow \mathbf{C}$ are in $\Lambda_{\alpha}$ then $f g \in \Lambda_{\alpha}$.

Proof. For $0 \leqslant k<\alpha<k+1 \in \mathbf{Z}$, the result is obvious by Leibniz's rule and induction on $k$. For $\alpha=1$, the result follows from the identity

$$
\begin{aligned}
f(x+h) g(x+h)+ & f(x-h) g(x-h)-2 f(x) g(x) \\
= & g(x-h)\{f(x+h)+f(x-h)-2 f(x)\} \\
& +f(x+h)\{g(x+h)+g(x-h)-2 g(x)\} \\
& -2\{f(x+h)-f(x)\}\{g(x-h)-g(x)\} .
\end{aligned}
$$

For $1<\alpha=k \in \mathbf{Z}$, the result follows by induction.

LEMMA 2.4. If $K \subseteq \mathbf{R}$ is a compact interval, $p$ is a polynomial on $\mathbf{R}$, and $f \in \Lambda_{\alpha}(\mathbf{R})$, then $p f \in \Lambda_{\alpha}(K)$.

PRoof. Apply the local variant of 2.3 .

Lemma 2.5. Let $f: \mathbf{R} \rightarrow \mathbf{C}, f \in \Lambda_{\alpha}(\mathbf{R}), 0<l<\alpha<l+1 \in \mathbf{Z}$. Let $0 \leqslant k<l$ be an integer and let $q \geqslant l$ be an integer. Then there exist constants $c(j, k, l, q)$, $0 \leqslant j \leqslant q$, such that for any $h \in \mathbf{R}, h \neq 0$,

$$
\sum_{j=0}^{q} c(j, k, l, q) f(x+(2 j-l) h)=h^{k} f^{(k)}+O\left(|h|^{\alpha}\right) .
$$

Proof. By Lemma 2.1,

$$
f(x+\eta)=f(x)+\eta f^{\prime}(x)+\cdots+\left(\eta^{l} / l !\right) f^{(l)}(x)+O\left(|\eta|^{\alpha}\right),
$$

any $\eta \in \mathbf{R}$. So

$$
\begin{aligned}
& \sum_{j=0}^{q} c(j, k, l, q) f(x+(2 j-l) h) \\
&=\sum_{s=0}^{l} \frac{f^{(s)}(x)}{s !} \sum_{j=0}^{q} c(j, k, l, q)((2 j-l) h)^{s}+O\left(|h|^{\alpha}\right) .
\end{aligned}
$$

Therefore proving the sublemma amounts to solving the system of $l+1$ equations

$$
\begin{gathered}
\sum_{j=0}^{q} c(j, k, l, q)((2 j-l) h)^{s}=0, \quad s=0,1, \ldots, k-1, k+1, \ldots, l, \\
\sum_{j=0}^{q} c(j, k, l, q)((2 j-l) h)^{k}=k !
\end{gathered}
$$

in the $(q+1)$ unknowns $c(0, k, l, q), \ldots, c(q, k, l, q)$. Since the matrix of coefficients is a Vandermonde matrix, the system may be solved.

Now we need some results from approximation theory. Let $\varphi \in C_{c}^{\infty}(\mathbf{R})$ be even with $\int \varphi d x=1$. Define $\varphi_{j}(x)=2^{j} \varphi\left(2^{j} x\right), j=0,1,2, \ldots$, and let $\psi_{j}(x)=\varphi_{j}(x)-$ $\varphi_{j-1}(x)$ when $j=1,2, \ldots ; \psi_{0}(x) \equiv \varphi_{0}(x)$. If $f \in L^{\infty}(\mathbf{R})$, define

$$
\psi_{j} f(x)=f * \psi_{j}(x), \quad \varphi_{j} f(x)=f * \varphi_{j}(x) .
$$

LEMMA 2.6. If $f \in \mathscr{B} \subseteq(\mathbf{R})$ then $\sum_{j=0}^{N} \psi_{j} f=\varphi_{N} f \rightarrow f$ uniformly on compact sets as $N \rightarrow \infty$. 
Proof. This is well known (see [18]).

LEMMA 2.7. If $f \in \Lambda_{\alpha}$ (R), $0 \leqslant k<\alpha<k+1 \in \mathbf{Z}$, then

$$
\begin{aligned}
\left\|\psi_{j} f\right\|_{C^{k}} & \leqslant C \cdot 2^{-j(\alpha-k)}\|f\|_{\Lambda_{\alpha}}, \\
\left\|\psi_{j} f\right\|_{C^{k+1}} & \leqslant C \cdot 2^{-j(\alpha-(k+1))}\|f\|_{\Lambda_{\alpha}}, \\
\left\|\varphi_{j} f\right\|_{C^{k}} & \leqslant C \cdot 2^{-j(\alpha-k)}\|f\|_{\Lambda_{\alpha}} .
\end{aligned}
$$

PROoF. Since convolution commutes with constant coefficient differential operators,

$$
\begin{aligned}
\left|\left(\frac{d}{d x}\right)^{k} \psi_{j} f(x)\right| & =\left|\int f^{(k)}(x-t) \psi_{j}(t) d t\right| \\
& =\left|\int\left\{f^{(k)}(x-t)-f^{(k)}(x)\right\} \psi_{j}(t) d t\right| \\
& \leqslant\|f\|_{\Lambda_{\alpha}} \int 2^{-j(\alpha-k)}\left|\psi_{j}(t)\right| d t<C 2^{-j(\alpha-k)}\|f\|_{\Lambda_{\alpha}},
\end{aligned}
$$

where we have used the fact that $\int \psi_{j} d x=0$. Similarly,

$$
\begin{aligned}
\left|\left(\frac{d}{d x}\right)^{k+1} \psi_{j} f(x)\right| & =\left|\int f^{(k)}(x-t) 2^{2 j} \psi_{0}^{\prime}\left(2^{j} t\right) d t\right| \\
& =2^{2 j}\left|\int\left\{f^{(k)}(x-t)-f^{(k)}(x)\right\} \psi_{0}^{\prime}\left(2^{j} t\right) d t\right| \\
& \leqslant 2^{2 j} 2^{-j(\alpha-k)}\|f\|_{\Lambda_{\alpha}} \int\left|\psi_{0}^{\prime}\left(2^{j} t\right)\right| d t \\
& \leqslant C 2^{-j(\alpha-(k+1))}\|f\|_{\Lambda_{\alpha}} .
\end{aligned}
$$

For the third inequality, notice that

$$
\begin{aligned}
\left\|\varphi_{j} f\right\|_{C^{k}} & \leqslant \sum_{l=j}^{\infty}\left\|\psi_{j} f\right\|_{C^{k}} \leqslant \sum_{l=j}^{\infty} C \cdot 2^{-l(\alpha-k)}\|f\|_{\Lambda_{\alpha}} \\
& \leqslant C \cdot 2^{-j(\alpha-k)}\|f\|_{\Lambda_{\alpha} \cdot \square} \square
\end{aligned}
$$

Lemma 2.8. Let $f \in \Lambda_{\alpha}(\mathbf{R}), \alpha \in \mathbf{Z}$. Let $0<\delta<1$. Then

$$
\begin{aligned}
& \left\|\psi_{j} f\right\|_{\Lambda_{\alpha+\delta}} \leqslant C \cdot 2^{j \delta}\|f\|_{\Lambda_{\alpha}}, \quad\left\|\psi_{j} f\right\|_{\Lambda_{\alpha-\delta}} \leqslant C \cdot 2^{-j \delta}\|f\|_{\Lambda_{\alpha}}, \\
& \left\|\varphi_{j} f\right\|_{\Lambda_{\alpha-\delta}} \leqslant C \cdot 2^{-j \delta}\|f\|_{\Lambda_{\alpha} \cdot}
\end{aligned}
$$

Proof. We attack this problem indirectly. Now

$$
\begin{aligned}
\left|(d / d x)^{\alpha-1} \psi_{j} f(x)\right| & =\left|\psi_{j} f^{(\alpha-1)}(x)\right| \\
& =\left|\int f^{(\alpha-1)}(x-t) \psi_{j}(t) d t\right| \\
& =\frac{1}{2}\left|\int\left\{f^{(\alpha-1)}(x-t)+f^{(\alpha-1)}(x+t)-2 f^{(\alpha-1)}(x)\right\} \psi_{j}(t) d t\right|
\end{aligned}
$$


because $\psi_{j}$ is even and has mean value zero. So this is majorized by

$$
\frac{1}{2}\|f\|_{\Lambda_{\alpha}} 2^{-j} \int\left|\psi_{j}(t)\right| d t \leqslant C 2^{-j}\|f\|_{\Lambda_{\alpha}} .
$$

Likewise,

$$
\begin{aligned}
& \left|\left(\frac{d}{d x}\right)^{\alpha+1} \psi_{j} f(x)\right|=\left|2^{3 j} \int f^{(\alpha-1)}(x-t) \psi^{\prime \prime}\left(2^{j} t\right) d t\right| \\
& \quad=\frac{1}{2} \cdot 2^{3 j}\left|\int\left\{f^{(\alpha-1)}(x-t)+f^{(\alpha-1)}(x+t)-2 f^{(\alpha-1)}(x)\right\} \psi^{\prime \prime}\left(2^{j} t\right) d t\right| \\
& \quad \leqslant C \cdot 2^{3 j} \cdot 2^{-j} \cdot\|f\|_{\Lambda_{\alpha}} \cdot \int\left|\psi^{\prime \prime}\left(2^{j} t\right)\right| d t,
\end{aligned}
$$

where we have used the fact that $\psi^{\prime \prime}$ is even and has mean value zero. The last line is now majorized by

$$
C \cdot 2^{j}\|f\|_{\Lambda_{a}}
$$

Now the first two estimates follow by complex or real interpolation from (2.8.1), (2.8.2). The last one follows by addition.

Corollary 2.9. Let $f \in \mathscr{B} \mathcal{C}(\mathbf{R})$. Let $f \in \Lambda_{\alpha}(\mathbf{R}), 0<k<\alpha<k+1 \in \mathbf{Z}$. Then $f \in \Lambda_{\alpha}(\mathbf{R})$ if and only if there is a $C>0$ so that for each $\lambda>1$ we can write $f$ as $f(x)=f^{0}(x)+f^{1}(x)$ with

$$
\left\|f^{0}\right\|_{C^{k}} \leqslant C \lambda^{k-\alpha}, \quad\left\|f^{1}\right\|_{C^{k+1}} \leqslant C \lambda^{k+1-\alpha} .
$$

Proof. Choose $N \in \mathbf{Z}$ with $\lambda<2^{N} \leqslant 2 \lambda$. Let $f \in \Lambda_{\alpha}(\mathbf{R})$. Define

$$
f^{0}=f-\sum_{j=0}^{N} \psi_{j} f=\sum_{j=N+1}^{\infty} \psi_{j} f, \quad f^{1}=\sum_{j=0}^{N} \psi_{j} f
$$

By Lemma 2.7,

$$
\begin{aligned}
\left\|f^{0}\right\|_{C^{k}} & \leqslant \sum_{j=N+1}^{\infty}\left\|\psi_{j} f\right\|_{C^{k}} \leqslant \sum_{j=N+1}^{\infty} C\|f\|_{\Lambda_{\alpha}} \cdot 2^{-j(\alpha-k)} \\
& \leqslant C \cdot\|f\|_{\Lambda_{\alpha}} 2^{-N(\alpha-k)} \leqslant C\|f\|_{\Lambda_{\alpha}} \cdot \lambda^{k-\alpha} .
\end{aligned}
$$

Also,

$$
\begin{aligned}
\left\|f^{1}\right\|_{C^{k+1}} & \leqslant \sum_{j=0}^{N}\left\|\psi_{j} f\right\|_{C^{k+1}} \leqslant \sum_{j=0}^{N} C\|f\|_{\Lambda_{\alpha}} 2^{-j(\alpha-(k+1))} \\
& \leqslant C\|f\|_{\Lambda_{\alpha}} \cdot 2^{-N(\alpha-(k+1))} \leqslant C\|f\|_{\Lambda_{\alpha}} \cdot \lambda^{k+1-\alpha} .
\end{aligned}
$$

Conversely, assume $f$ has the indicated decomposition for every $\lambda$. Let $h \in \mathbf{R}$, $0<|h|<1$. Then

$$
\begin{aligned}
\left|\Delta_{h}^{k+1} f(x)\right| & \leqslant\left|\Delta_{h}^{k+1} f^{0}(x)\right|+\left|\Delta_{h}^{k+1} f^{1}(x)\right| \\
& \leqslant C|h|^{k}\left\|f^{0}\right\|_{C^{k}}+C|h|^{k+1}\left\|f^{1}\right\|_{C^{k+1}}
\end{aligned}
$$

where $\lambda$ is at our disposal. The last line is

$$
\leqslant C \lambda^{k-\alpha}|h|^{k}+C \lambda^{k+1-\alpha}|h|^{k+1} \text {. }
$$


Now let $\lambda=1 /|h|$ to obtain

$$
\left|\Delta_{h}^{k+1} f(x)\right| \leqslant C|h|^{\alpha}
$$

so (since $f$ is bounded) $f \in \Lambda_{\alpha}(\mathbf{R})$.

Corollary 2.10. Let $f \in \mathscr{B} C(\mathbf{R})$ and $\alpha \in \mathbf{Z}$. Let $0<\delta<1$. Then $f \in \Lambda_{\alpha}(\mathbf{R})$ if and only if there is a $C=C(\delta)$ so that for every $\lambda \geqslant 1$ we can write $f$ as $f=f^{0}+f^{1}$ with

$$
\left\|f^{0}\right\|_{\Lambda_{\alpha-\delta}} \leqslant C \cdot \lambda^{-\delta}, \quad\left\|f^{1}\right\|_{\Lambda_{\alpha+\delta}} \leqslant C \cdot \lambda^{\delta} .
$$

Proof. Similar to 2.9 .

3. The principal argument for the a priori estimate, $\alpha \notin Z$. In this section we derive an a priori estimate which amounts to the assertion of the Main Theorem for functions in $\mathscr{B} \bigodot \cap C^{\infty}$. We restrict attention to $\alpha \notin \mathbf{Z}$. Later the case $\alpha \in \mathbf{Z}$ will be derived from this one.

The proof of the a priori estimate breaks into two parts, which we formulate as Lemmas A and B (resp. B') below and prove in $\$ \S 4$ and 5. We first introduce some notation.

If $\mathrm{g}$ is a stratified Lie algebra, $\mathrm{g}=V_{1} \oplus \cdots \oplus V_{m}$, let $\left\{X_{1}, \ldots, X_{n}\right\}$ be a basis for $V_{1}$. If $1 \leqslant j \in \mathbf{Z}$, let

$$
\mathscr{g}_{j}=\left\{\left(i_{1}, \ldots, i_{j}\right): i_{l} \in\{1, \ldots, n\}, \text { all } 1 \leqslant l \leqslant j\right\} .
$$

If $I \in q_{j}$, let

$$
V_{j} \ni X_{I} \equiv\left[X_{i_{1}},\left[X_{i_{2}},\left[\cdots\left[X_{i_{j-1}}, X_{i_{j}}\right] \cdots\right]\right]\right] .
$$

Obviously $\left\{X_{I}\right\}_{I \in g_{j}}$ spans $V_{j}, 1 \leqslant j \leqslant m$. If $0<\alpha<\infty, I \in \mathscr{G}_{j}$, set $\alpha\left(X_{I}\right)=\alpha(I)$ $=\alpha(1-j / \alpha)$ (we will introduce a variant of this notation in Lemma $\left.B^{\prime}\right)$. If $\mathscr{X}=\left\{X_{I_{1}}, \ldots, X_{I_{p}}\right\}, I_{l} \in \mathcal{G}_{j_{l}}$, set $\gamma(\mathcal{X})=1-\sum_{l=1}^{p} j_{l} / \alpha$.

Since only Lipschitz norms (and an occasional sup norm) are considered here, we use \|\|$_{\alpha}$ to denote \|\|$_{\Lambda_{\alpha}}$. If \|\|$_{\beta}$ ever appears with $\beta \leqslant 0$, then the term is understood to be zero.

If $f: G \rightarrow C, \beta>0,0 \neq X \in \mathfrak{g}$, then let

$$
\|f\|_{X, \beta} \equiv \sup _{g \in G}\|f(g \exp X \cdot)\|_{\beta} .
$$

This notation will occur repeatedly in the sequel. Also, the letters $C, C^{\prime}$, etc. will denote many different constants whose values may change from line to line.

LEMMA A. Let $G$ be a stratified group, $g=V_{1} \oplus \cdots \oplus V_{m}$ its Lie algebra, and let $\alpha_{1}, \alpha_{2}>0, \alpha_{j} \notin \mathbf{Z}$. Define $1 / \beta \equiv 1 / \alpha_{1}+1 / \alpha_{2}$ and assume $\beta \notin \mathbf{Z}$. Let $A_{1} \in$ $V_{j_{1}}, A_{2} \in V_{j_{2}}, 1 \leqslant j_{1}, j_{2} \leqslant m$, and let $B=\left[A_{1}, A_{2}\right]$. Then for any $f \in \mathscr{B} \bigodot \cap$ $C^{\infty}(G)$,

$$
\|f\|_{B, \beta} \leqslant C\left\{\|f\|_{A_{1}, \alpha_{1}}+\|f\|_{A_{2}, \alpha_{2}}\right\} .
$$

The constant $C$ depends on $\alpha_{1}, \alpha_{2}, m$ but not on $A_{1}, A_{2}$ nor $f$. 
LEMMA B. Let $G$ be a stratified group, $\mathrm{g}=V_{1} \oplus \cdots \oplus V_{m}$ its Lie algebra, and let $0<\alpha \notin \mathbf{Z}$. Let $\left\{X_{1}, \ldots, X_{n}\right\}$ be a vector space basis for $V_{1}$. If $f \in \mathscr{B} \subset \cap C^{\infty}$ then for any $\mathcal{X}=\left\{X_{I_{1}}, \ldots, X_{I_{p}}\right\}, I^{\prime} \in \mathscr{G}_{k}$ it holds that

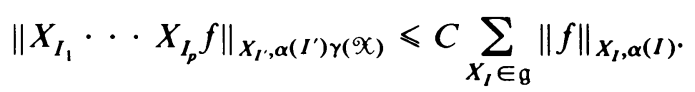

Here $C$ depends on $\alpha, m$ but not on $f$ nor on the choice of $\left\{X_{1}, \ldots, X_{n}\right\}$.

The following is a generalization of Lemma B which is rather more technical but is easier to prove inductively.

LEMMA $\mathrm{B}^{\prime}$. Let $G$ be a stratified group, $\mathfrak{g}=V_{1} \oplus \cdots \oplus V_{m}$ its Lie algebra, and let $\alpha_{1}, \ldots, \alpha_{n}>0, \alpha_{j} \notin \mathbf{Z}$. If $I \in g_{j}$ let $\alpha(I)$ be defined by

$$
1 / \alpha(I)=1 / \alpha_{i_{1}}+\cdots+1 / \alpha_{i_{j}} \text {. }
$$

Assume $\alpha(I) \notin \mathbf{Z}$ for all $I \in \cup_{j=1}^{m} g_{j}$. Then for any $f \in \mathscr{B} \bigodot \cap C^{\infty}$, any $\mathcal{X}=$ $\left\{X_{I_{1}}, \ldots, X_{I_{p}}\right\} \subseteq \mathrm{g}$, any $X_{I}^{\prime} \in \mathfrak{g}$,

$$
\left\|X_{I_{1}} \cdots X_{I_{p}} f\right\|_{X_{I}, \alpha\left(I^{\prime}\right)\left(1-\Sigma 1 / \alpha\left(I_{I}\right)\right)} \leqslant C \sum_{X_{I} \in \mathfrak{g}}\|f\|_{X_{I}, \alpha(I)} .
$$

Assuming these lemmas, we now supply the

Proof of THE A PRIORI eSTIMATE WHEN $\alpha \notin Z$. Let $G$ be a fixed stratified group, $\mathrm{g}=V_{1} \oplus \cdots \oplus V_{m}$ its Lie algebra. Let $\left\{X_{1}, \ldots, X_{n}\right\}$ be a fixed vector space basis for $V_{1}$. Let the constant $C_{0}$ be as in the statement of the Main Theorem.

Now for any $I \in g_{2}$, Lemma $A$ implies that $\|f\|_{X_{I}, \alpha / 2} \leqslant C_{0}$. Inductively, if it has been shown that

$$
\|f\|_{X_{I}, \alpha / j} \leqslant C \cdot C_{0}, \quad \text { all } I \in g_{j}, \quad 1 \leqslant j \leqslant j_{0},
$$

let $I^{\prime} \in g_{j_{0}+1}$. Then $X_{I^{\prime}}=\left[X_{i_{1}}, X_{l}\right]$, some $I \in Q_{j_{0}}$. Thus Lemma A applies with $\alpha_{1}=\alpha, \alpha_{2}=\alpha / j_{0}, \beta=\alpha /\left(j_{0}+1\right)$. So

$$
\|f\|_{X_{I}, \alpha /\left(j_{0}+1\right)} \leqslant C \cdot C_{0} \text {. }
$$

Therefore, by induction, (3.1) holds for all $j, 1 \leqslant j \leqslant m$. By Lemma B, it follows that for any $\mathcal{X}=\left\{X_{I_{1}}, \ldots, X_{I_{p}}\right\}, I^{\prime} \in \mathscr{G}_{k}, f \in \mathscr{B} \bigodot \cap C^{\infty}$,

$$
\left\|X_{I_{1}} \cdots X_{I_{p}} f\right\|_{X_{I}, \alpha \gamma(x) / k} \leqslant C \cdot C_{0} \text {. }
$$

Recall that we are assuming $\alpha \notin \mathbf{Z}$. Therefore statement (iii) of the Main Theorem is unchanged if we assume $\alpha \gamma / l<1$, for this may be achieved by adding $[\alpha \gamma / l]$ $Z$ 's to the monomial $Y_{1} \cdots Y_{k}$. This having been noted, statements (ii) and (iii) of the Main Theorem follow since each $Y_{i} \in V_{j(i)}$ is a linear combination of $\left\{X_{I}\right\}_{I \in g_{j(i)}}$. Statement (i) follows trivially from (iii) and the triangle inequality.

Thus, modulo Lemmas A and B, we have proved the desired $a$ priori estimate.

4. The proof of Lemma A. It is convenient to specialize to the (stratified) Lie algebra $\mathfrak{h}$ generated by $\left\{A_{1}, A_{2}\right\}$. Let $V_{j}^{\prime} \subseteq \mathfrak{h}$ denote the linear span of the $(j-1)$ th order commutators of $A_{1}, A_{2}$. Certainly $V_{j}^{\prime}=0$ for $j>m^{\prime}+1$, some $m^{\prime} \leqslant m$. So $\mathfrak{h}=V_{1}^{\prime} \oplus \cdots \oplus V_{m^{\prime}}^{\prime}$ is a stratification. Let $\mathfrak{h}_{j}=V_{j}^{\prime} \oplus \cdots \oplus V_{m^{\prime}}^{\prime}$. 
If $I=\left(i_{1}, \ldots, i_{k}\right) \in\{1,2\} \times \cdots \times\{1,2\}$, let

$$
A_{I}=\left[A_{i_{1}},\left[A_{i_{2}},\left[\cdots\left[A_{i_{k-1}}, A_{i_{k}}\right] \cdots\right]\right]\right] .
$$

Let $1 / \alpha\left(A_{I}\right) \equiv 1 / \alpha(I) \equiv \sum_{l=1}^{k} 1 / \alpha_{i}$. If $\mathbb{Q}=\left\{A_{I_{1}}, \ldots, A_{I_{p}}\right\}$, let $\gamma(\mathbb{Q})=1-$ $\Sigma 1 / \alpha\left(A_{I_{l}}\right)$.

It should be noted that Lemma $\mathrm{B}^{\prime}$ is used to prove Lemma $\mathrm{A}$.

Lemma 4.1. Let $1 \leqslant l<\alpha_{1}, l \in \mathbf{Z}$. Then either $l>\beta$ or $\left(1-l / \alpha_{1}\right) \alpha_{2}>\beta$.

Proof. If not then both $l \leqslant \beta$ and $\left(1-l / \alpha_{1}\right) \alpha_{2} \leqslant \beta$ so

$$
\beta=\alpha_{1} \alpha_{2} /\left(\alpha_{1}+\alpha_{2}\right)=\left(\alpha_{1}-\beta\right) \alpha_{2} / \alpha_{1} \leqslant\left(\alpha_{1}-l\right) \alpha_{2} / \alpha_{1} \leqslant \beta .
$$

It follows that the inequalities are equalities, so $\beta=l \in \mathbf{Z}$. This contradicts the assumption that $\beta \notin \mathbf{Z}$.

Lemma 4.2. Let $1 \leqslant l<\alpha_{1}, l \in \mathbf{Z}$. Then

$$
\left\|\left(A_{1}\right)^{l} f\right\|_{A_{2}, \alpha_{2}\left(1-l / \alpha_{1}\right)} \leqslant c\|f\|_{B, \beta}+C\left\{\|f\|_{A_{1}, \alpha_{1}}+\|f\|_{A_{2}, \alpha_{2}}+\sup _{A_{I} \in \mathfrak{h}_{3}}\|f\|_{A_{t}, \alpha(I)}\right\}
$$

for all $f \in \mathscr{B} C \cap C^{\infty}$. Here $c$ is a constant which may be made arbitrarily small as long as $C$ is large enough. The choice of $C=C(c)$ does not depend on $f$.

Proof. By Lemma 4.1 there are two cases.

If $\left(1-l / \alpha_{1}\right) \alpha_{2}>\beta$ then apply Lemma $B^{\prime}$ with $g$ replaced by $\mathfrak{h}$ and $\left\{X_{1}, \ldots, X_{n}\right\}$ replaced by $\left\{A_{1}, \lambda A_{2}\right\}$. Here $\lambda \geqslant 1$ is a constant to be selected. Write $A_{1}^{\prime}=A_{1}, A_{2}^{\prime}=\lambda A_{2}, B^{\prime}=\left[A_{1}^{\prime}, A_{2}^{\prime}\right]=\lambda B$. Define $A_{1}^{\prime}$ similarly. Then Lemma $\mathrm{B}^{\prime}$ yields

$$
\left\|\left(A_{I}^{\prime}\right)^{l} f\right\|_{A_{2}^{\prime}, \alpha_{2}\left(1-l / \alpha_{1}\right)} \leqslant C\left\{\|f\|_{A_{2}^{\prime}, \alpha_{2}}+\|f\|_{A^{\prime}, \alpha_{1}}+\|f\|_{B^{\prime}, \beta}+\max _{A_{l}^{\prime} \in \mathfrak{h}_{3}}\|f\|_{A_{i}^{\prime}, \alpha\left(A_{i}^{\prime}\right)}\right\} .
$$

If we write out this inequality with the dependence on $\lambda$ made explicit, then we obtain

$$
\begin{aligned}
& \lambda^{\left(1-l / \alpha_{1}\right) \alpha_{2}}\left\|\left(A_{1}\right)^{l} f\right\|_{A_{2}, \alpha_{2}\left(1-l / \alpha_{1}\right)} \\
& \leqslant C\left\{\lambda^{\alpha_{2}}\|f\|_{A_{2}, \alpha_{2}}+\|f\|_{A_{1}, \alpha_{1}}+\lambda^{\beta}\|f\|_{B, \beta}+\sup _{A_{I} \in \mathfrak{h}_{3}} \lambda^{i(I)}\|f\|_{A_{t}, \alpha\left(A_{I}\right)}\right\} .
\end{aligned}
$$

Here $i(I)$ is the number of occurrences of the digit 2 in $I$. Now divide through by $\lambda^{\left(1-l / \alpha_{1}\right) \alpha_{2}}$ and choose $\lambda$ so large that $C \lambda^{\beta-\left(1-l / \alpha_{1}\right) \alpha_{2}} \leqslant c$.

The case $l>\beta$ is handled similarly: let $A_{1}^{\prime}=\lambda A_{1}, A_{2}^{\prime}=A_{2}$, and argue as before.

LEMMA 4.3. For all $f \in \mathscr{B} \subseteq \cap C^{\infty}$ it holds that

$$
\begin{aligned}
\|f\|_{B, \beta} \leqslant & C^{\prime}\|f\|_{A_{1}, \alpha_{1}}+C^{\prime}\|f\|_{A_{2}, \alpha_{2}}+C^{\prime} \max _{1<l<\alpha_{1}}\left\|A_{1}^{l} f\right\|_{A_{2}, \alpha_{2}\left(1-l / \alpha_{1}\right)} \\
& +C^{\prime} \max _{\substack{\mathcal{Q}=\left\{A_{I_{1}}, \ldots, A_{l_{p}}\right\} \subseteq \mathfrak{h}_{3} \cup\left\{A_{2}\right\} \\
p>1}}\left\|A_{I_{1}} \cdots A_{I_{p}} f\right\|_{B, \beta \gamma(\mathbb{Q})} .
\end{aligned}
$$


Proof. Let $0 \leqslant r_{j}<\alpha_{j}<r_{j}+1 \in \mathbf{Z}, j=1,2$. Let $0<b<\beta<b+1 \in \mathbf{Z}$. Let $g \in G, h, k \in \mathbf{R}$. For $0 \leqslant t \leqslant r_{2}$ write, using Lemma 2.1 ,

$$
f\left(g \exp \left(2 t-r_{2}\right) h A_{2} \exp k A_{1}\right)=\sum_{q=0}^{r_{1}} \frac{k^{q} A_{1}^{q} f\left(g \exp \left(2 t-r_{2}\right) h A_{2}\right)}{q !}+O\left(|k|^{\alpha_{1}}\right) .
$$

Summing over $t$ with coefficients

$$
(-1)^{r_{2}+t}\left(\begin{array}{c}
r_{2} \\
t
\end{array}\right)
$$

yields

$$
\begin{aligned}
\sum_{t=0}^{r_{2}}(-1)^{r_{2}}+t\left(\begin{array}{c}
r_{2} \\
t
\end{array}\right) f\left(g \exp \left(2 t-r_{2}\right) h A_{2} \exp k A_{1}\right) \\
=\sum_{t=0}^{r_{2}}(-1)^{r_{2}+t}\left(\begin{array}{c}
r_{2} \\
t
\end{array}\right) \sum_{q=0}^{r_{1}} k^{q} A_{1}^{q} f\left(g \exp \left(2 t-r_{2}\right) h A_{2}\right) / q !+O\left(|k|^{\alpha_{1}}\right) .
\end{aligned}
$$

We apply the Campbell-Hausdorff formula to write the $t$ th term on the left as

$$
\begin{aligned}
& (-1)^{r_{2}+t}\left(\begin{array}{c}
r_{2} \\
t
\end{array}\right) f\left(g \exp k A_{1} \exp -h k\left(2 t-r_{2}\right) B \exp \left(2 t-r_{2}\right) h A_{2}\right.
\end{aligned}
$$

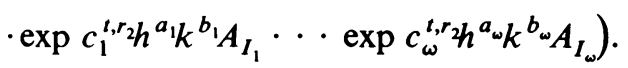

Here each $A_{I_{l}} \in \mathfrak{h}_{3}$ and $a_{l}, b_{l} \in \mathbf{N}, c_{l}^{t, r_{2}} \in \mathbf{Q}$ (see Hochschild [10] for details on the Campbell-Hausdorff formula; the values of these constants are of no interest here-only that $c_{l}^{t, r_{2}}$ depends polynomially on $t, r_{2}$ and not on $\left.f, g, A_{1}, A_{2}\right)$. Of course the Campbell-Hausdorff formula is an infinite asymptotic expansion. It terminates after finitely many terms in the present case because $\mathrm{g}$ is nilpotent. Now substitute (4.3.2) into (4.3.1), each $t$, and replace $g$ by $g$ exp $-k A_{1}$. So

$$
\begin{aligned}
& \sum_{t=0}^{r_{2}}(-1)^{r_{2}+t}\left(\begin{array}{c}
r_{2} \\
t
\end{array}\right) f\left(g \exp -h k\left(2 t-r_{2}\right) B \exp \left(2 t-r_{2}\right) h A_{2}\right.
\end{aligned}
$$

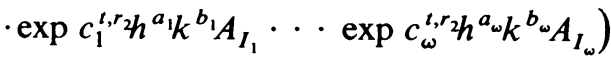

$$
\begin{aligned}
& =\left.\sum_{q=0}^{r_{1}} \frac{k^{q}}{q !} \Delta_{h}^{r_{2}}\left(A_{1}^{q} f\left(g \exp -k A_{1} \exp A_{2} \cdot\right)\right)\right|_{0}+O\left(|k|^{\alpha_{1}}\right) \text {. }
\end{aligned}
$$

Apply Lemma 2.1 repeatedly to each term on the left of (4.3.3). The $t$ th term then becomes

$$
\begin{array}{r}
(-1)^{r_{2}+t}\left(\begin{array}{c}
r_{2} \\
t
\end{array}\right)\left\{f\left(g \exp -h k\left(2 t-r_{2}\right) B\right)+\left(2 t-r_{2}\right) h A_{2} f\left(g \exp -h k\left(2 t-r_{2}\right) B\right)\right. \\
\left.+\cdots+\left(\left(2 t-r_{2}\right)^{r_{2}} h^{r_{2}} / r_{2} !\right) A_{2}^{r_{2}} f\left(g \exp -h k\left(2 t-r_{2}\right) B\right)\right\} \\
+O\left(|h|^{\alpha_{2}}\right)+\sum \text { (higher order terms) }+O\left(|h|^{\alpha_{2}}+|k|^{\alpha_{1}}\right),
\end{array}
$$

where the higher order terms are of the form

$$
e_{\gamma}^{t} h^{\gamma}\left(2 t-r_{2}\right)^{\gamma} A_{2}^{\gamma}\left(c_{\mu}^{t, s} h^{a_{\mu}} k^{b_{\mu}} A_{I_{\mu}}\right)^{\eta_{\mu}} \ldots\left(c_{\omega}^{t, r_{2}} h^{a_{\omega}} k^{b_{\omega}} A_{I_{\omega}}\right)^{\eta_{\omega}} f\left(g \exp -h k\left(2 t-r_{2}\right) B\right),
$$


$0 \leqslant \gamma \leqslant r_{2}, 1 \leqslant \mu \leqslant \omega, 0 \leqslant \eta_{l}<\alpha\left(I_{l}\right)$. Now substitute (4.3.4) into (4.3.3) to obtain

$$
\begin{aligned}
\left.\sum h^{a_{i}^{\prime}} k^{b_{i}} \Delta_{h k}^{r_{2}} A_{2}^{\alpha} p_{l}(\cdot) A_{I_{\mu_{l}}} \cdots A_{I_{\omega_{l}}} f(g \exp B \cdot)\right|_{0} \\
=\left.\sum_{q=0}^{r_{1}} \frac{k^{q}}{q !} \Delta_{h}^{r_{2}}\left(A_{1}^{q} f\left(g \exp -k A_{1} \exp A_{2} \cdot\right)\right)\right|_{0}+O\left(|k|^{\alpha_{1}}\right) .
\end{aligned}
$$

Here the $p_{l}$ are polynomials. Recall from (4.3.4) that one summand on the left side of (4.3.5) is

$$
\left.\Delta_{h k}^{r_{2}} f(g \exp B \cdot)\right|_{0} .
$$

Isolate this term on the left of (4.3.5), set $h \in \theta^{\alpha_{1}}, k=\theta^{\alpha_{2}}$, and use Lemmas 2.2 and 2.4 , to estimate the remaining terms to obtain the result.

LEMMA 4.4. For all $f \in \mathscr{B} \bigodot \cap C^{\infty}$ it holds that

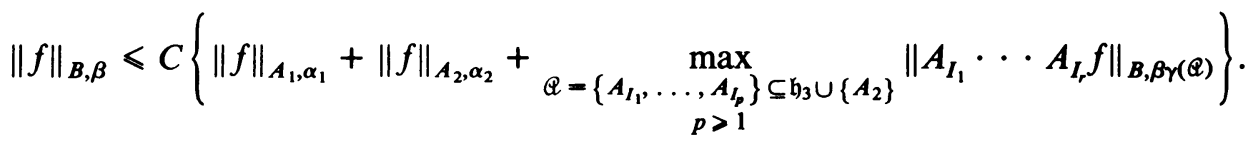

Proof. Substitute the result of Lemma 4.2 into the right side of Lemma 4.3, choosing $c$ so small that $c C^{\prime}=\frac{1}{2}$. Now transpose the term $\frac{1}{2}\|f\|_{B, \beta}$ to the left side of the inequality.

LEMMA 4.5. For all $f \in \mathscr{B} \bigodot \cap C^{\infty}$ it holds that

$$
\begin{aligned}
\|f\|_{B, \beta} \leqslant & c\|f\|_{A_{2}, \alpha_{2}} \\
& +C^{\prime \prime}\left\{\|f\|_{A_{1}, \alpha_{1}}+\underset{\mathcal{Q}=\left\{A_{I_{1}}, \ldots, A_{I_{p}}\right\} \subseteq \mathfrak{h}_{3} \cup\left\{A_{2}\right\}}{ }\left\|A_{I_{1}} \cdots A_{I_{p}} f\right\|_{B, \beta \cdot \gamma(\mathcal{Q})}\right\} .
\end{aligned}
$$

Here c can be made arbitrarily small if $C^{\prime \prime}$ is made sufficiently large.

Proof. The proof is the same as that of 4.2 (except that now there is but one case). Set $A_{1}^{\prime}=\lambda A_{1}, A_{2}^{\prime}=A_{2}$. Apply Lemma 4.4, and choose $\lambda$ large.

LEMMA 4.6. For any $\mathscr{Q}=\left\{A_{I_{1}}, \ldots, A_{I_{r}}\right\} \subseteq \mathfrak{h}_{3} \cup\left\{A_{2}\right\}$, all $f \in \mathscr{B} \subset \cap C^{\infty}$, it holds that

$$
\left\|A_{I_{1}} \cdots A_{I_{r}} f\right\|_{B, \beta \gamma(\mathcal{Q})} \leqslant c^{\prime}\|f\|_{B, \beta}+C\left\{\|f\|_{A_{2}, \alpha_{2}}+\max _{A_{I} \in \mathfrak{h}_{3}}\|f\|_{A_{l}, \alpha(I)}\right\} .
$$

Here $c^{\prime}$ can be made arbitrarily small so long as $C$ is sufficiently large.

Proof. First apply Lemma $B^{\prime}$ to the Lie algebra generated by $\left\{A_{I_{1}}, \ldots, A_{I_{p}}, A_{2}, B\right\}$ to obtain

$$
\left\|A_{I_{1}} \cdots A_{I_{p}} f\right\|_{B, \beta \gamma(\mathcal{Q})} \leqslant C\left\{\|f\|_{B, \beta}+\|f\|_{A_{2}, \alpha_{2}}+\max _{A_{I} \in \mathfrak{h}_{3}}\|f\|_{A_{I}, \alpha(I)}\right\} .
$$

Now the crucial fact here is that the constant $C$ in (4.6.1) depends on $m$ and $p$ but not on the choice of $A_{I_{1}}, \ldots, A_{I_{p}}, B, A_{2}$. In particular, (4.6.1) persists with $A_{I_{j}}^{\prime} \equiv$ $\lambda A_{I,}, j=1, \ldots, p, A_{2}^{\prime}=\lambda A_{2}$, and with $B$ unchanged-with the same constant $C$. Now the proof is concluded by writing explicitly the dependence on $\lambda$, dividing through by $\lambda^{p}$, and choosing $\lambda$ large. 
LEMMA 4.7. For all $f \in \mathscr{B} \bigodot \cap C^{\infty}$ it holds that

$$
\|f\|_{B, \beta} \leqslant c^{\prime \prime}\|f\|_{A_{2}, \alpha_{2}}+C\left\{\|f\|_{A_{1}, \alpha_{1}}+\max _{A_{I} \in \mathfrak{h}_{3}}\|f\|_{A_{I}, \alpha(I)}\right\},
$$

where $c^{\prime \prime}$ may be made arbitrarily small if $C$ is chosen sufficiently large.

Proof. Substitute Lemma 4.6 into the right-hand side of Lemma 4.5, choosing $c^{\prime}=c^{\prime \prime} /(2 C)$. Now transpose the term $\frac{1}{2}\|f\|_{B, \beta}$ to the left side.

Proof of Lemma A. By Lemma 4.7, write

$$
\begin{aligned}
\|f\|_{B, \beta} & \leqslant C\left\{\|f\|_{A_{1}, \alpha_{1}}+\|f\|_{A_{2}, \alpha_{2}}+\sum_{A_{I} \in \mathfrak{h}_{3}}\|f\|_{A_{l}, \alpha(I)}\right\} \\
& =C\left\{\|f\|_{A_{1}, \alpha_{1}}+\|f\|_{A_{2}, \alpha_{2}}+\sum_{A_{I} \in V_{3}^{\prime}}+\cdots+\sum_{A_{1} \in V_{m^{\prime}}^{\prime}}\right\} .
\end{aligned}
$$

If $A_{I} \in V_{3}^{\prime}$, then $A_{I}= \pm\left[A_{i}, B\right], i=1$ or 2 . Apply Lemma 4.7 with $\left\{A_{1}, A_{2}\right\}$ replaced by $A_{i}, B$ to obtain

$$
\|f\|_{A_{I}, \alpha(I)} \leqslant c\|f\|_{B, \beta}+C\left\{\|f\|_{A_{i}, \alpha_{i}}+\max _{A_{J} \in \mathfrak{h}_{4}}\|f\|_{A_{j}, \alpha(I)}\right\} .
$$

For each $A_{I} \in \mathfrak{h}_{3}$, plug this into (*), with $c$ sufficiently small, and transpose the $\|f\|_{B, \beta}$ term to the left side to obtain

$$
\|f\|_{B, \beta} \leqslant C\left\{\|f\|_{A_{1}, \alpha_{1}}+\|f\|_{A_{2}, \alpha_{2}}+\sum_{A_{I} \in V_{4}^{\prime}}+\cdots+\sum_{A_{I} \in V_{m^{\prime}}^{\prime}}\right\} .
$$

This process may be repeated $m^{\prime}-4$ more times to complete the proof.

5. The proof of Lemma $\mathbf{B}^{\prime}$. We introduce the notation $\mathfrak{g}_{j}=V_{j} \oplus \cdots \oplus V_{m}$, $j=1, \ldots, m$. We shall prove, by backwards induction on $j \in\{1, \ldots, m\}$, the statement

Let $\alpha_{1}, \ldots, \alpha_{n}>0, \alpha_{j} \notin \mathbf{Z}$. Then, for any function $f \in$ $C^{\infty}(G)$, it holds that

$$
\max _{\substack{X_{I} \in \mathfrak{g} \\ \mathcal{X}=\left\{X_{I_{1}}, \ldots, X_{l_{p}}\right\} \subseteq g_{j}}}\left\|X_{I_{1}} \cdots X_{I_{p}} f\right\|_{X_{I}, \alpha(I) \gamma(\mathcal{X})} \leqslant C \max _{X_{I} \in \mathfrak{g}}\|f\|_{X_{I}, \alpha(I)} \equiv C \cdot \mathcal{C} .
$$

In case $j=m$, the Lie algebra elements $X_{I_{1}}, \ldots, X_{I_{p}}, X_{I}$ commute. So, for $g \in G$ fixed, the function

$$
F\left(t_{1}, \ldots, t_{p}, s\right)=f\left(g \exp t_{i} X_{I_{1}} \cdots \exp t_{p} X_{I_{p}} \exp s X_{I}\right)
$$

satisfies

$$
\begin{gathered}
\sup _{t_{1}, \ldots, t_{l-1}, t_{l+1}, \ldots, t_{p}, s} \| F\left(t_{1}, \ldots, t_{l-1}, \cdot, t_{l+1}, \ldots, t_{p}, s \|_{\alpha\left(I_{l}\right)}\right)<C \cdot C, \\
\sup _{t_{1}, \ldots, t_{p}}\left\|F\left(t_{1}, \ldots, t_{p}, \cdot\right)\right\|_{\alpha(I)}<C \cdot C .
\end{gathered}
$$

By the classical theory for functions on $\mathbf{R}^{p+1}$ (see [12]), it follows that

$$
\sup _{t_{1}, \ldots, t_{p}}\left\|\left(\frac{\partial}{\partial t_{1}}\right) \cdots\left(\frac{\partial}{\partial t_{p}}\right) F\left(t_{1}, \ldots, t_{p}, \cdot\right)\right\|_{\alpha(I) \gamma(\mathscr{X})}<C \cdot \mathcal{C} .
$$


In other words, since the estimate is uniform in $g$,

$$
\left\|X_{I_{1}} \cdots X_{I_{p}} f\right\|_{X_{I}, \alpha(I) \gamma(\mathcal{X})}<C \mathcal{C} .
$$

So $S_{m}$ holds.

Now suppose inductively that $S_{j+1}, \ldots, S_{m}$ have been proved. Let $X_{\mathrm{I}_{p}} \in \mathrm{g}_{j}$ and let $X_{I} \in \mathrm{g}$ be arbitrary. Let $r<\alpha(I)<r+1 \in \mathbf{Z}, q<\alpha\left(I_{p}\right)<q+1 \in \mathbf{Z}$. Let $h, h_{p} \in \mathbf{R}, 0 \leqslant t \leqslant r+1$. Apply Lemma 2 with $\alpha$ replaced by $\alpha\left(I_{p}\right), q$ by $q+1, l$ by $q, k=1, f$ by $f\left(g \exp (2 t-(r+1)) h X_{I} \exp X_{\mathrm{I}_{p}} \cdot\right)$. So

$$
\begin{aligned}
\sum_{j=0}^{q+1} c(j, 1, q, q+ & 1) f\left(g \exp (2 t-(r+1)) h X_{I} \exp (2 j-(q+1)) h_{p} X_{I_{p}}\right) \\
& =h_{p}\left(X_{I_{p}} f\right)\left(g \exp (2 t-(r+1)) h X_{1}\right) .
\end{aligned}
$$

Multiply both sides by $(-1)^{t+r+1}\left(\begin{array}{c}r+1 \\ t\end{array}\right)$ and sum over $t$ to obtain

$$
\begin{gathered}
\sum_{j=0}^{q+1} c(j, 1, q, q+1) \sum_{t=0}^{r+1}(-1)^{t+r+1}\left(\begin{array}{c}
r+1 \\
t
\end{array}\right) \\
\cdot f\left(g \exp (2 t-(r+1)) h X_{I} \exp (2 j-(q+1)) h_{p} X_{I_{p}}\right) \\
=\left.h_{p} \Delta_{h}^{r+1}\left(X_{I_{p}} f\right)\left(g \exp X_{I} \cdot\right)\right|_{0} .
\end{gathered}
$$

We apply the Campbell-Hausdorff formula to rewrite the $t$ th term of the inner sum on the left side as

$$
\begin{aligned}
(-1)^{t+r+1}\left(\begin{array}{c}
r+1 \\
t
\end{array}\right) f\left(g \exp (2 j-(q+1)) h_{p} X_{I_{p}} \exp (2 t-(r+1)) h X_{I}\right. \\
\left.\cdot \exp (2 t-(r+1))(2 j-(q+1)) h h_{p}\left[X_{I}, X_{I_{p}}\right] \exp \cdots\right)
\end{aligned}
$$

where $\cdots$ denotes terms involving higher commutators of $X_{I}, X_{I_{p}}$. As in (4.3.2), the asymptotic expansion provided by the Campbell-Hausdorff formula terminates after finitely many terms because $g$ is nilpotent. Notice that $\left[X_{I}, X_{I_{p}}\right] \in g_{j+1}$ so that the inductive hypothesis may be applied to this expression if it can be appropriately isolated. But repeated application of Lemma 2.1 enables us to write (5.2) as

$$
\begin{aligned}
(-1)^{t+r+1}\left(\begin{array}{c}
r+1 \\
t
\end{array}\right)\{ & f\left(g \exp (2 j-(q+1)) h_{p} X_{I_{p}} \exp (2 t-(r+1)) h X_{I}\right) \\
& +h h_{p}(2 j-(q+1))(2 t-(r+1))\left(\left[X_{I}, X_{I_{p}}\right] f\right) \\
& \cdot\left(g \exp (2 j-(q+1)) h_{p} X_{I_{p}} \exp (2 t-(r+1)) h X_{I}\right)
\end{aligned}
$$

+ higher order terms

where the higher order terms involve derivatives of $f$ by monomials consisting of elements of $g_{j+1}$. Now we set $h=\theta^{1 / \alpha(I)}, h_{p}=\theta^{1 / \alpha\left(I_{p}\right)}$, plug (5.3) into (5.1) and estimate using 2.2 and 2.4 (just as in the proof of 4.3 ) to obtain

$$
\left|\theta^{1 / \alpha\left(I_{p}\right)} \Delta_{\theta^{1 / \alpha(I)}}^{r+1}\left(X_{I_{p}} f\right)\left(g \exp X_{I} \cdot\right)\right|_{0} \mid \leqslant C \cdot \mathcal{C} \cdot \theta
$$

or

$$
\left|\Delta_{\theta^{\prime}}^{r+1}\left(X_{I_{p}} f\right)\left(g \exp X_{I} \cdot\right)\right|_{0} \mid \leqslant C \cdot \mathcal{C} \cdot\left(\theta^{\prime}\right)^{\alpha(I)\left(1-1 / \alpha\left(I_{p}\right)\right)}
$$

whence

$$
\left\|X_{I_{p}} f\right\|_{X_{I}, \alpha(I)\left(1-1 / \alpha\left(I_{p}\right)\right)} \leqslant C \cdot \mathcal{C}
$$


Notice that inequality (5.4) holds for $X_{I_{p}} \in g_{j}$ and any $X_{I} \in \mathfrak{g}$. Now we apply this argument again, with $I_{p-1}$ replacing $I_{p}, X_{I_{p}} f$ replacing $f$, to obtain

$$
\left\|X_{I_{p-1}} X_{I_{p}} f\right\|_{X_{l}, \alpha(I)\left(1-1 / \alpha\left(I_{p-1}\right)-1 / \alpha\left(I_{p}\right)\right)}<C \max _{X_{J} \in g}\left\|X_{I_{p}} f\right\|_{X_{J}, \alpha(J)\left(1-1 / \alpha\left(I_{p}\right)\right)}
$$

By (5.4), this last line does not exceed $C \mathcal{C}$. The argument may now be repeated $p-2$ more times to obtain the full statement of $S_{j}$. This completes the induction, and the proof of $\mathbf{B}^{\prime}$.

6. Some lemmas of Friedrichs type about convolution smoothing. Naturally, the passage from the $a$ priori estimates to the full result is effected via Friedrichs mollifiers. In this section we isolate some estimates which are variants of the classical Friedrichs Lemma. Some of these will not be needed until $\S \S 8,9$.

Let $G$ be identified with its underlying Euclidean space $\mathbf{R}^{N}$ via the exponential map. Haar measure on $G$ is just Lebesgue measure, denoted $d x$. All results in this section, and all integrals, are in the standard Euclidean structure. To facilitate this, vector fields are written $\sum_{j=1}^{N} a_{j}(x)\left(\partial / \partial x_{j}\right)$.

Now fix a $\Phi \in C_{c}^{\infty}\left(\mathbf{R}^{N}\right), \Phi>0, \int \Phi d x=1$. For $\varepsilon>0$ define $\Phi_{\varepsilon}(x)=$ $\varepsilon^{-N} \Phi(x / \varepsilon)$. If $f \in L_{\text {loc }}^{1}\left(\mathbf{R}^{N}\right)$, let

$$
f_{\varepsilon}(x)=f * \Phi_{\varepsilon}(x)=\int_{\mathbf{R}^{N}} f(x-t) \Phi_{\varepsilon}(t) d t .
$$

Then it is known (see [18]) that $f_{\varepsilon} \rightarrow f$ a.e. If $f$ is continuous then $f_{\varepsilon} \rightarrow f$ uniformly on compact sets.

Lemma. 6.1. Let $f \in \mathscr{B} \mathcal{C}(G), X \in \mathfrak{g}, 0 \leqslant k \in \mathbf{Z}$, and suppose that

$$
\sup _{g \in G}\|f(g \exp X \cdot)\|_{C^{k}(\mathbf{R})} \equiv C_{k}<\infty \text {. }
$$

Then for any compact $E \subset G$,

$$
\sup _{g \in E}\left\|f_{\varepsilon}(g \exp X \cdot)\right\|_{C^{k}(\mathbf{R})} \leqslant C \cdot C_{k}
$$

where the constant $C$ is independent of $f$ and $\varepsilon$ but will depend on $E$.

Proof. Using a cutoff function, we may assume that $f$ is supported in a compact neighborhood $U$ of $O$. Write the vector field generated by $X$ as $\sum_{j=1}^{N} a_{j}(x)\left(\partial / \partial x_{j}\right)$. Of course the $a_{j}$ have bounded continuous derivatives of all orders on $U$. Now

$$
\begin{aligned}
X f_{\varepsilon}= & X \int \Phi_{\varepsilon}(x-t) f(t) d t=\sum \int a_{l}(x) \frac{\partial}{\partial x_{l}} \Phi_{\varepsilon}(x-t) f(t) d t \\
= & -\sum \int a_{l}(x)\left(\frac{\partial}{\partial t_{l}} \Phi_{\varepsilon}(x-t)\right) f(t) d t \\
= & -\sum \int a_{l}(t)\left(\frac{\partial}{\partial t_{l}} \Phi_{\varepsilon}(x-t)\right) f(t) d t+\sum \int O(\varepsilon)\left(\frac{\partial}{\partial t_{l}} \Phi_{\varepsilon}(x-t)\right) f(t) d t \\
= & -\sum \int a_{l}(t)\left(\frac{\partial}{\partial t_{l}} \Phi_{\varepsilon}(x-t)\right) f(t) d t \\
& +\sum \varepsilon^{-N} \int \alpha_{l}(t)\left(\frac{\partial}{\partial t_{l}} \Phi\right)\left(\frac{x-t}{\varepsilon}\right) f(t) d t \equiv \mathrm{I}+\mathrm{II},
\end{aligned}
$$


where the $\alpha_{l}$ are smooth and bounded with smooth bounded derivatives of all orders.

By integration by parts,

$$
I=\sum \int \Phi_{\varepsilon}(x-t) a_{l}(t) \frac{\partial}{\partial t_{l}} f(t) d t+\sum \int \Phi_{\varepsilon}(x-t) \tilde{\alpha}_{l}(t) f(t) d t .
$$

Hence

$$
\begin{aligned}
X f_{e} & =\mathrm{I}+\mathrm{II}=\Phi_{e} * X f+\sum \varepsilon^{-N} \int \Psi_{k}\left(\frac{x-t}{\varepsilon}\right) \beta_{k}(t) f(t) d t . \\
& \equiv A_{1}+B_{1} .
\end{aligned}
$$

Here the $\Psi_{k}, \beta_{k}, k=1, \ldots, 2 N$, are smooth, bounded functions with smooth, bounded derivatives. (5.1.1) implies, in particular, that

$$
\begin{aligned}
\left\|X f_{e}\right\|_{\text {sup }} & \leqslant\left\|\Phi_{e} * X f\right\|_{\text {sup }} A_{2}, \alpha_{2}+\left\|\sum\right\|_{\text {sup }} \\
& \leqslant\left\|\Phi_{e}\right\|_{L^{1}}\|X f\|_{\text {sup }}+\sum\left\|\Psi_{k}\right\|_{L^{1}}\left\|\beta_{k}\right\|_{\text {sup }}\|f\|_{\text {sup }} \\
& \leqslant C \sup _{g}\|f(g \exp X \cdot)\|_{C^{1}}=C \cdot C_{1} .
\end{aligned}
$$

To establish the result when $k=2$, apply $X$ to (5.1.1). So

$$
X^{2} f_{\varepsilon}=X A_{1}+X B_{1} \text {. }
$$

But the case $k=1$ may be applied $a$ priori to $A_{1}$ giving

$$
X A_{1}=\Phi_{e} * X^{2} f+\sum \varepsilon^{-N} \int \Psi_{k}\left(\frac{x-t}{\varepsilon}\right) \beta_{k}(t) X f(t) d t .
$$

Both of the terms on the right-hand side of this expression may be estimated by $C \sup _{g}\|f(g \exp X \cdot)\|_{C^{k}}$. On the other hand,

$$
\begin{aligned}
X A_{2}= & \sum \varepsilon^{-N} \sum \int a_{l}(x) \frac{\partial}{\partial x_{l}}\left(\Psi_{k}\left(\frac{x-t}{\varepsilon}\right)\right) \beta_{k}(t) f(t) d t \\
= & -\sum \varepsilon^{-N} \sum \int a_{l}(t) \frac{\partial}{\partial t_{l}}\left(\Psi_{k}\left(\frac{x-t}{\varepsilon}\right)\right) \beta_{k}(t) f(t) d t \\
& +\sum \varepsilon^{-N} \sum \int O(\varepsilon) \frac{\partial}{\partial t_{l}}\left(\Psi_{k}\left(\frac{x-t}{\varepsilon}\right)\right) \beta_{k}(t) f(t) d t \\
= & \text { etc., }
\end{aligned}
$$

and the proof is completed as in the case $k=1$.

A simple repetition of this argument yields the full result.

Lemma 6.2. Let $f \in \mathscr{B} \mathcal{C}(G), \quad 0 \neq X \in \mathfrak{g}, \quad 0<\alpha<\infty$. Suppose that $\sup _{g \in G}\|f(g \exp X \cdot)\|_{\alpha}=C_{\alpha}<\infty$. Then for any compact $E \subset G$,

$$
\sup _{g \in E}\left\|f_{e}(g \exp X \cdot)\right\|_{\alpha} \leqslant C \cdot C_{\alpha}
$$

where $C$ is independent of $0<\varepsilon<1$.

Proof. We use the techniques of real interpolation theory. Assume as in 6.1 that $f$ is compactly supported. For any function $g$, let $g_{e}=g * \Phi_{\varepsilon}$. First let $k<\alpha<k$ $+1 \in \mathbf{Z}$. We apply Corollary 2.9 along each trajectory of $X$ as follows. With $\varphi_{j}, \psi_{j}$ 
defined on $\mathbf{R}^{1}$ as in the discussion preceding $2.6, f \in \mathscr{B} \mathcal{C}$, compactly supported in a fixed $U \subset \subset G$, let

$$
f_{j}(g)=\int_{\mathbf{R}} f(g \exp X t) \psi_{j}(t) d t .
$$

Then for $\lambda>1$, let $N \sim \log _{2} \lambda$ as in the proof of 2.9 , and set

$$
f^{0}=f-\sum_{0}^{N} f_{j}, \quad f^{1}=\sum_{0}^{N} f_{j} .
$$

An argument like that in 2.9 shows that

$$
\begin{gathered}
\sup _{g}\left\|f^{0}(g \exp X \cdot)\right\|_{C^{k}} \leqslant C\|f\|_{\alpha} \lambda^{k-\alpha}, \\
\sup _{g}\left\|f^{1}(g \exp X \cdot)\right\|_{C^{k+1}}<C\|f\|_{\alpha} \lambda^{k+1-\alpha} .
\end{gathered}
$$

If $g \in G, 0 \neq h \in \mathbf{R}$, we have

$$
\begin{aligned}
\left|X^{k} f_{\varepsilon}(g \exp X h)-X^{k_{\varepsilon}}(g)\right| \leqslant & \left|X^{k}\left(f^{0}\right)_{\varepsilon}(g \exp X h)-X^{k}\left(f^{0}\right)_{e}(g)\right| \\
& +\left|X^{k}\left(f^{1}\right)_{\varepsilon}(g \exp h X)-X^{k}\left(f^{1}\right)_{\varepsilon}(g)\right| \\
\leqslant & 2 \sup \left|X^{k}\left(f^{0}\right)_{e}\right|+|h| \sup \left|X^{k+1}\left(f^{1}\right)_{\varepsilon}\right| \\
\leqslant & 2 C \lambda^{k-\alpha}+C \cdot|h| \cdot \lambda^{k+1-\alpha} .
\end{aligned}
$$

Now $\lambda$ is at our disposal, so let $\lambda=1 /|h|$. Thus

$$
\left|X^{k} f_{e}(g \exp X h)-X^{k} f_{e}(g)\right|<C|h|^{\alpha-k}
$$

as desired. The constants all depend on $U$.

The case $\alpha \in \mathbf{Z}$ may now be derived from the case $\alpha \notin \mathbf{Z}$ using 2.10.

7. Completion of the proof in case $\alpha \notin \mathrm{Z}$ : passing from a priori estimates to the full result. Let $f: G \rightarrow \mathbf{C}$ satisfy the hypotheses of the Main Theorem for $0<\alpha<$ $\infty, \alpha \notin \mathbf{Z}$. There is no loss to assume that $f$ is compactly supported. Let $\Phi, \Phi_{e}, f_{e}$ be as in the last section. Then by $6.2, f_{\varepsilon}$ satisfies the hypotheses of the Main Theorem with $C_{0}$ replaced by $C \cdot C_{0}, C$ independent of $\varepsilon$. Also $f_{\varepsilon} \rightarrow f$ uniformly on compact sets. By the a priori estimate, if $X_{I} \in \mathrm{g}$ then for any $g \in G, 0<h \in \mathbf{R}, \alpha<k \in \mathbf{Z}$,

$$
\left|\Delta_{h}^{k} f_{\varepsilon}\left(g \exp X_{I} \cdot\right)\right|_{0} / h^{\alpha(I)} \mid<C \cdot C_{0} .
$$

Letting $\varepsilon \rightarrow 0$ yields

$$
\left|\Delta_{h}^{k} f\left(g \exp X_{I} \cdot\right)\right|_{0} / h^{\alpha(I)} \mid \leqslant C \cdot C_{0},
$$

or, taking the sup over $g$,

$$
\|f\|_{X_{I}, \alpha(I)} \leqslant C \cdot C_{0}
$$

If $\alpha(I)>1$ this says that $X_{I} f$ exists. Of course $X_{I} f$ is the pointwise limit of $X_{I} f_{e}$. If $X_{J} \in \mathrm{g}$ is arbitrary then the a priori estimates yield that for $g \in G, 0<h \in \mathbf{R}$, $\alpha<k \in \mathbf{Z}$,

$$
\left|\Delta_{h}^{k} X_{I} f_{\varepsilon}\left(g \exp X_{J} \cdot\right)\right|_{0} / h^{\alpha(J)(1-1 / \alpha(I))} \mid \leqslant C \cdot C_{0}
$$

Letting $\varepsilon \rightarrow 0$ yields

$$
\left|\Delta_{h}^{k} X_{I} f\left(g \exp X_{J} \cdot\right)\right|_{0} / h^{\alpha(J)(1-\alpha(I))} \mid<C \cdot C_{0},
$$


or, taking the sup over $g$,

$$
\left\|X_{I} f\right\|_{X_{J}, \alpha(J)(1-1 / \alpha(I))} \leqslant C \cdot C_{0} .
$$

Repeating this argument we may verify all the conclusions of the Main Theorem.

8. The case $\alpha \in \mathbf{Z}$. The proof proceeds by methods of real interpolation, though there does not seem to be any way to apply real interpolation directly. Fix $0<\alpha \in \mathbf{Z}$. By induction arguments used before (especially at the end of $\S 5$ ), it is enough to consider vector fields two at a time: in other words, we may take $V_{1}$ to be two dimensional, with given basis $\left\{X_{1}, X_{2}\right\}$. In any case, this extra hypothesis simplifies the notation, not the proof. The result will follow from

LEMMA 8.1. There is $a C>0$ so that for every $0<\varepsilon<1$ and every $f \in \mathscr{B} \subset \cap$ $C^{\infty}$ it is possible to write $f=f^{0}+f^{1}$ with

$$
\begin{aligned}
& \left\|f^{0}\right\|_{X_{j}, \alpha-1 / 3} \leqslant C \cdot \varepsilon \cdot K(f), \quad j=1,2 ; \\
& \left\|f^{1}\right\|_{X_{j}, \alpha+1 / 3} \leqslant C \cdot \varepsilon^{-1} \cdot K(f), \quad j=1,2 .
\end{aligned}
$$

Here

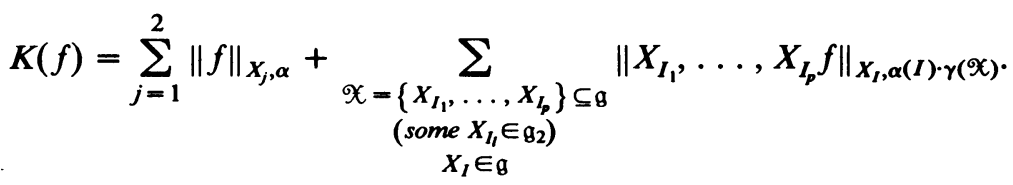

The constant $C$ depends only on $m, \alpha$.

Let us see how the Main Theorem follows, assuming 8.1. To do so, we first derive from 8.1 a modification thereof:

LEMMA 8.2. There is a $C>0$ so that for any $0<\varepsilon<1$, any $\eta>1$, any $f \in \mathscr{B} \bigodot \cap C^{\infty}$, it is possible to write $f=f^{0}+f^{1}$ with

$$
\begin{aligned}
& \left\|f^{0}\right\|_{X_{1}, \alpha-1 / 3}<C \cdot \varepsilon \cdot K_{\eta}(f), \\
& \left\|f^{1}\right\|_{X_{1}, \alpha+1 / 3}<C \cdot \varepsilon^{-1} \cdot K_{\eta}(f), \\
& \left\|f^{0}\right\|_{X_{2}, \alpha-1 / 3}<C(\eta) \cdot \varepsilon \cdot K(f), \\
& \left\|f^{1}\right\|_{X_{2}, \alpha+1 / 3}<C(\eta) \cdot \varepsilon^{-1} \cdot K(f) .
\end{aligned}
$$

Here $K(f)$ is as in the preceding lemma, $C(\eta)$ is a possibly large constant which depends on $\eta$, and

$$
\begin{gathered}
K_{\eta}(f)=\eta \cdot\|f\|_{X_{1}, \alpha}+\eta^{-1}\|f\|_{X_{2}, \alpha} \\
+\eta^{-1} \sum_{\substack{x=\left\{X_{I_{1}}, \ldots, X_{L_{p}}\right\} \\
\left(\operatorname{some} X_{I_{l}} \in \mathfrak{g}_{2}\right) \\
X_{I} \in g}}\left\|X_{I_{1}} \cdots X_{I_{p}} f\right\|_{X_{I}, \alpha(I) \cdot \gamma(x)} .
\end{gathered}
$$

The constant $C$ depends only on $m, \alpha$. 
Proof. Apply Lemma 8.1 to the vector fields $X_{1}^{\prime}=\lambda X_{1}, X_{2}^{\prime}=X_{2}$. Express the dependence on $\lambda$ explicitly:

$$
\begin{aligned}
& \lambda^{\alpha-1 / 3}\left\|f^{0}\right\|_{X_{1}, \alpha-1 / 3} \\
& \leqslant C \varepsilon\left\{\lambda^{\alpha}\|f\|_{X_{1}, \alpha}+\|f\|_{X_{2}, \alpha}\right. \\
& \left.\quad+\sum \lambda^{i\left(I_{1}\right)+\cdots+i\left(I_{p}\right)+\alpha(I) \gamma(X) i(I) / j(I)}\left\|X_{I_{1}} \cdots X_{I_{p}} f\right\|_{X_{1}, \alpha(I) \cdot \gamma(X)}\right\},
\end{aligned}
$$

where $i(J)$ denotes the number of occurrences of the digit 1 in the index $J$ and $j(I)$ is the unique $j$ such that $X_{I} \in V_{j}$. The condition that some $X_{I_{1}} \in g_{2}$ guarantees that the exponents ocurring in the sum are less than $\alpha-1 / 3$. So if we divide through by $\lambda^{\alpha-1 / 3}$ and choose $\lambda=\lambda(\eta)$ large enough then (8.2.1) follows. The other estimates are computed in the same way (from the same dilation of $X_{1}$ !).

By the usual technique of dilating $X_{1}$ and leaving $X_{2}$ fixed we may prove

LEMMA 8.3. For $0<\beta \notin \mathbf{Z}$ the a priori estimate holds in the form

$$
\sum_{\substack{\left.X_{I_{1}}, \ldots, X_{I_{p}}\right\} \subseteq \mathrm{g} \\ X_{I} \in g}}\left\|X_{I_{1}} \cdots X_{I_{p}} f\right\|_{X_{1}, \beta(I) \gamma(\mathcal{X})} \leqslant c\|f\|_{X_{2}, \beta}+C\|f\|_{X_{1}, \beta}
$$

where $c$ may be made arbitrarily small simply by choosing $C$ large enough. Here $\Sigma^{*}$ denotes summation over $X_{I_{1}}, \ldots, X_{I_{p}}, X_{I}$ which are not all equal to $X_{2}$.

Now we will derive an a priori estimate for the Main Theorem for $\alpha \in \mathbf{Z}$ by applying the a priori estimate for $\alpha-\frac{1}{3}, \alpha+\frac{1}{3}$ in the form 8.3 to the elements of the decomposition in 8.2. More precisely, let $l=\alpha+1,0<h \in \mathbf{R}$, choose $X_{J} \in \mathfrak{g}$, $X_{J} \neq X_{2}, g \in G$, and estimate

$$
\left.\left.\left|\Delta_{h}^{l} f\left(g \exp X_{J} \cdot\right)\right|_{0}|\leqslant| \Delta_{h}^{l} f^{0}\left(g \exp X_{J} \cdot\right)\right|_{0}|+| \Delta_{h}^{l} f^{1}\left(g \exp X_{J} \cdot\right)\right|_{0} \mid
$$

where $f^{0}, f^{1}$ are as in 8.2 and $\varepsilon, \eta$ are at our disposal. Now the last line is

$$
\begin{aligned}
\leqslant & |h|^{(\alpha-1 / 3) / j(J)}\left\|f^{0}\right\|_{X_{J},(\alpha-1 / 3) / j(J)} \\
& +|h|^{(\alpha+1 / 3) / j(J)}\left\|f^{1}\right\|_{X_{J},(\alpha+1 / 3) / j(J)} \\
\leqslant & |h|^{(\alpha-1 / 3) / j(J)}\left[c\left\|f^{0}\right\|_{X_{2}, \alpha-1 / 3}+C\left\|f^{0}\right\|_{X_{1}, \alpha-1 / 3}\right] \\
& +|h|^{(\alpha+1 / 3) / j(J)}\left[c\left\|f^{1}\right\|_{X_{2}, \alpha+1 / 3}+C\left\|f^{1}\right\|_{X_{1}, \alpha+1 / 3}\right] \\
\leqslant & |h|^{(\alpha-1 / 3) / j(J)}\left[c C(\eta) \varepsilon K(f)+C \cdot \varepsilon K_{\eta}(f)\right] \\
& +|h|^{(\alpha+1 / 3) / j(J)}\left[c C(\eta) \varepsilon^{-1} K(f)+C \cdot \varepsilon^{-1} K_{\eta}(f)\right] .
\end{aligned}
$$

Let $\varepsilon=|h|^{1 /(3 j(J))}$ and take the supremum over $g \in G$ to obtain

$$
\|f\|_{X_{J}, \alpha(J)} \leqslant 2 c C(\eta) K(f)+2 C K_{\eta}(f) .
$$

Now sum over $J$ to get

$$
\sum_{\substack{X_{J} \in \mathfrak{g} \\ X_{J} \neq X_{2}}}\|f\|_{X_{J}, \alpha(J)} \leqslant 2 c C(\eta) K(f)+2 C K_{\eta}(f)
$$


Now iterative arguments as in the end of $\$ 7$ give, in fact,

$$
\sum_{\substack{\left\{X_{I_{1}}, \ldots, X_{I_{p}}\right\} \\ X_{I} \in g}}\left\|X_{I_{1}} \cdots X_{I_{p}} f\right\|_{X_{I}, \alpha(I) \gamma(\mathscr{x})} \leqslant C^{\prime}\left[2 c C(\eta) K(f)+2 C K_{\eta}(f)\right] .
$$

If $\eta$ is first chosen large enough, then $c$ is chosen small enough, we obtain

$$
\Sigma^{*} \leqslant C\left(\|f\|_{X_{1}, \alpha}+\|f\|_{X_{2}, \alpha}\right)+\frac{1}{2} \Sigma^{*}
$$

or

$$
\sum \leqslant 2 C\left(\|f\|_{X_{1}, \alpha}+\|f\|_{X_{2}, \alpha}\right)
$$

This is the desired a priori estimate. The full result may now be obtained precisely as in $\S 7$.

It remains to prove Lemma 8.1. We will need both isotropic and nonisotropic mollifiers. Let $\varphi, \psi, \varphi_{j}, \psi_{j}$ be as in $\S 2$. Let $\Phi, \Phi_{\varepsilon}$ be as in $\S 6$. If $f \in \mathscr{B} \mathcal{C}, X \in \mathfrak{g}$, $g \in G$ write

$$
\begin{aligned}
& \varphi_{j}^{X} f(g)=\int_{\mathbf{R}} f(g \exp t X) \varphi_{j}(t) d t=\int_{\mathbf{R}} f\left(\exp 2^{-j} t X\right) \varphi(t) d t \\
& \psi_{j}^{X} f(g)=\int_{\mathbf{R}} f(g \exp t X) \psi_{j}(t) d t=\int_{\mathbf{R}} f\left(g \exp 2^{-j} t X\right) \psi(t) d t
\end{aligned}
$$

Let

$$
f_{j}=\varphi_{j}^{X} \varphi_{j}^{X}\left(\Phi_{2^{-j}} * f\right)
$$

where $j$ will be selected at our convenience.

Our basic tool will be the notation

$$
\text { ad } X(Y)=[X, Y], \quad X, Y \in \mathfrak{g}
$$

and the following lemma of [11, p. 165]:

LEMmA 8.4. Let $f \in \mathscr{B} \bigodot \cap C^{\infty}, X, Y \in \mathrm{g}$. Then

$$
X \varphi_{j}{ }^{Y} f=\int\left(e^{-2^{-j} \text { ad } Y} X f\right)\left(g \exp 2^{-j} t Y\right) \varphi(t) d t
$$

Proof. Apply the Campbell-Hausdorff formula.

LEMMA 8.5. Let $f \in \mathscr{B} \bigodot \cap C^{\infty}$. Let $X_{1}, X_{2}$ be the usual basis vectors for $V_{1}$. Let $0 \leqslant k \in \mathbf{Z}$. Then

$$
\sup _{g}\left\|\left(\varphi_{j}^{X_{1}}\left(\Phi_{2^{-j}} * f\right)\left(g \exp X_{2} \cdot\right)\right)\right\|_{C^{k}}
$$

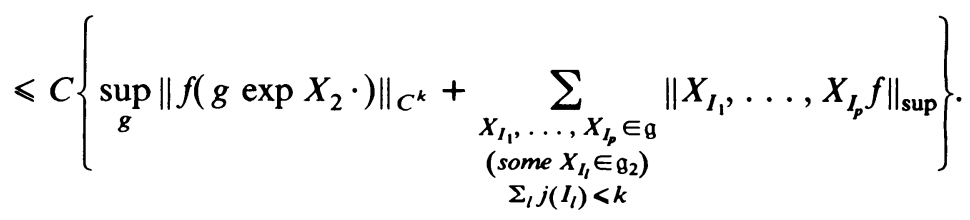


Proof. We may assume $k \geqslant 1$. By 8.4 ,

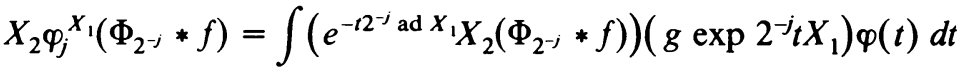

$$
\begin{aligned}
& =\sum_{l=0}^{k-1} \int\left(\frac{\left(-t 2^{-j} \operatorname{ad} X_{1}\right)^{l}}{l !} X_{2}\left(\Phi_{2^{-j}} * f\right)\right)\left(g \exp 2^{-j} t X_{1}\right) \varphi(t) d t \\
& +\int t^{k} 2^{-j k} Z\left(\Phi_{2^{-j}} * f\right)\left(g \exp 2^{-j} t X_{1}\right) \varphi(t) d t \quad\left(\text { where } Z \in V_{k+1}\right) \\
& \equiv T+R \text {. }
\end{aligned}
$$

Notice that the first term in $T$ is $\varphi_{j}^{X_{1}} X_{2} \Phi_{2^{-j}} * f$. The others involve derivatives of $f$ of the type occurring in the sum on the right side of (8.5.1). (We are using 6.1 here). Finally,

$$
|R| \leqslant 2^{-j} 2^{j} C\|f\| \sup \leqslant C \cdot\|f\|_{\text {sup }}
$$

because we cause the $Z$ differentiation to land on the $\Phi_{2^{-j}}$. Successive applications of $X_{2}$ to (8.5.2), together with (8.4), yield the result.

Lemma 8.6. Let $f \in \Re \bigodot \cap C^{\infty}$. Let $X_{1}, X_{2}$ be as in 8.5. Let $0<\alpha<\infty$. Then

$$
\left\|\varphi_{j} X_{1}\left(\Phi_{2^{-j}} * f\right)\right\|_{X_{2}, \alpha} \leqslant\left\{\|f\|_{X_{2}, \alpha}+\sum_{\substack{X=\left\{X_{I_{1}}, \ldots, X_{I_{p}}\right\} \\\left(\operatorname{some} X_{I_{1}} \in \mathfrak{g}_{2}\right) \\ X_{I} \in g}}\left\|X_{I_{1}}, \ldots, X_{I_{p}} f\right\|_{X_{l}, \alpha(I) \gamma(\mathfrak{X})}\right\} .
$$

Proof. First assume that $k<\alpha<k+1 \in \mathbf{Z}$. For every $0<\varepsilon<1$, use 2.9 to write $f=f^{0}+f^{1}$ where

$$
\begin{gathered}
\sup _{g}\left\|f^{0}\left(g \exp X_{2} \cdot\right)\right\|_{C^{k}} \leqslant C\|f\|_{X_{1}, \alpha} \cdot \varepsilon^{\alpha-k}, \\
\sup _{g}\left\|f^{1}\left(g \exp X_{2} \cdot\right)\right\|_{C^{k+1}} \leqslant C\|f\|_{X_{2}, \alpha} \cdot \varepsilon^{\alpha-(k+1)} .
\end{gathered}
$$

Then apply 8.5 to $f^{0}, f^{1}$ and choose $\varepsilon$ adroitly (as usual).

Now if $0<\alpha \in \mathbf{Z}$, use 2.10 to write, for every $0<\varepsilon<1, f=f^{0}+f^{1}$ with

$$
\left\|f^{0}\right\|_{X_{2}, \alpha-1 / 3} \leqslant C \cdot \varepsilon\left\|f^{0}\right\|_{X_{2}, \alpha}, \quad\left\|f^{1}\right\|_{X_{2}, \alpha+1 / 3}<C \cdot \varepsilon^{-1}\|f\|_{X_{2}, \alpha} .
$$

Applying the result from the first part of the proof to $f^{0}, f^{1}$, choosing $\varepsilon$ appropriately.

Lemma 8.7. Let $f \in \mathscr{B} \bigodot \cap C^{\infty}$. For $1 \leqslant j \in \mathbf{Z}$, let $f_{j}$ be as in the discussion preceding 8.4. Let $f^{0}=f-f_{N}, f^{1}=f_{N}$. Let

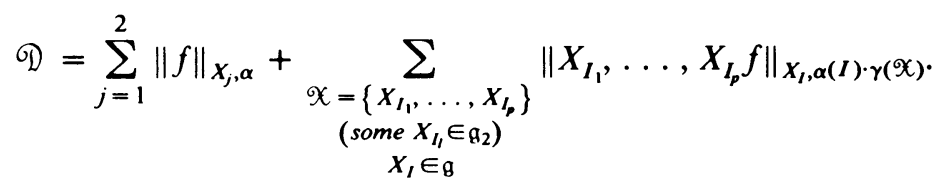

Then

$$
\begin{aligned}
& \left\|f^{0}\right\|_{X_{1}, \alpha-1 / 3} \leqslant C \cdot \mathscr{D} \cdot 2^{-N / 3}, \quad\left\|f^{0}\right\|_{X_{2}, \alpha-1 / 3}<C \cdot \mathscr{D} \cdot 2^{-N / 3}, \\
& \left\|f^{1}\right\|_{X_{1}, \alpha+1 / 3} \leqslant C \cdot \mathscr{D} \cdot 2^{N / 3}, \quad\left\|f^{1}\right\|_{X_{2}, \alpha+1 / 3} \leqslant C \cdot \mathscr{D} \cdot 2^{N / 3} .
\end{aligned}
$$


Proof. We may assume $\mathscr{D}=1$. Write

$$
f^{1}=\sum_{j=0}^{N} \psi_{j}^{X} \varphi_{N}^{X_{2}} \Phi_{2^{-N}} * f
$$

By 8.6 and 6.2,

$$
\left\|\varphi_{N}^{X_{2}} \Phi_{2^{-N}} * f\right\|_{X_{1}, \alpha}<C .
$$

So, by 2.8 ,

$$
\left\|\psi_{j}^{X} \varphi_{N}^{X} \Phi_{2^{-N}} * f\right\|_{X_{1}, \alpha+1 / 3} \leqslant C \cdot 2^{j / 3}
$$

Thus

$$
\left\|f^{1}\right\|_{X_{1}, \alpha+1 / 3} \leqslant \sum_{j=0}^{N} C \cdot 2^{j / 3} \leqslant C \cdot 2^{N / 3} .
$$

Likewise, we can write

$$
f^{1}=\sum_{j=0}^{N} \varphi_{N}^{X} \psi_{j}^{X} \Phi_{2^{-N}} * f
$$

Then, by 2.8 and 6.2 ,

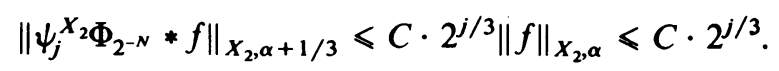

So a variant of 8.6 implies

$$
\left\|\varphi_{N}^{X} \psi_{j}^{X} \Phi_{2^{-N}} * f\right\|_{X_{2}, \alpha+1 / 3} \leqslant C \cdot 2^{j / 3} .
$$

Therefore

$$
\left\|f^{1}\right\|_{X_{2}, \alpha+1 / 3} \leqslant \sum_{j=0}^{N} C \cdot 2^{j / 3} \leqslant C \cdot 2^{N / 3} .
$$

Now $f^{0}$ is handled similarly: write

$$
\begin{aligned}
f^{0} & =f-\varphi_{N}^{X} \varphi_{N}^{X} \Phi_{2^{-N}} * f \\
& =\sum_{j=N+1}^{\infty} \varphi_{j}^{X} \varphi_{j} \varphi_{2} \Phi_{2^{-j}} * f-\varphi_{j-1}^{X} \varphi_{j-1}^{X_{2}} \Phi_{2^{-j+1}} * f .
\end{aligned}
$$

But

$$
\left\|\varphi_{j}^{X_{2}} \Phi_{2^{-j}} * f\right\|_{X_{2, \alpha-1 / 3}} \leqslant C \cdot 2^{-j / 3}
$$

by 2.8 whence, by a variant of 8.6 ,

$$
\left\|\varphi_{j}^{X} \varphi_{j}^{X_{2}} \Phi_{2^{-j}} * f\right\|_{X_{2, \alpha-1 / 3}} \leqslant C \cdot 2^{-j / 3} .
$$

As a result,

$$
\left\|f^{0}\right\|_{X_{2, \alpha-1 / 3}} \leqslant \sum_{j=N+1}^{\infty} C \cdot 2^{-j / 3} \leqslant C \cdot 2^{-N / 3} .
$$

The estimate for $\left\|f^{0}\right\|_{X_{1}, \alpha-1 / 3}$ is handled similarly.

Notice that 8.1 now follows from 8.7 by choosing $N \sim 3 \log 1 / \varepsilon$. This completes the discussion of the case $\alpha \in \mathbf{Z}$. 
9. Results in other norms. It is worth noting that variants of the Lipschitz spaces may be defined as follows. Fix $1 \leqslant p<\infty$. Let

$$
\begin{aligned}
& \Re_{\alpha}^{p}\left(\mathbf{R}^{N}\right)=\left\{f \in L^{p}\left(\mathbf{R}^{N}\right): \sup _{h \in \mathbf{R}^{N}}\|f(\cdot+h)-f(\cdot)\|_{L^{p}\left(\mathbf{R}^{N}\right)} /|h|^{\alpha}\right. \\
& \left.+\|f\|_{L^{p}\left(\mathbf{R}^{N}\right)} \equiv\|f\|_{\mathscr{T}_{\alpha}^{p}}<\infty\right\}, \quad 0<\alpha<1 ; \\
& \Re_{1}^{p}\left(\mathbf{R}^{N}\right)=\left\{f \in L^{p}\left(\mathbf{R}^{N}\right): \sup _{h \in \mathbf{R}^{N}} \frac{\|f(\cdot+h)-f(\cdot-h)-2 f(\cdot)\|_{L^{p}\left(R^{N}\right)}}{|h|}+\|f\|_{L^{p}\left(\mathbf{R}^{N}\right)} \equiv\|f\|_{\Re_{1}^{p}}<\infty\right\} ; \\
& \Re_{\alpha}^{p}\left(\mathbf{R}^{N}\right)=\left\{f \in L^{p}\left(\mathbf{R}^{N}\right): \sum_{j=1}^{N}\left\|\frac{\partial f}{\partial x_{j}}\right\|_{\mathscr{T}_{\alpha-1}^{p}}+\|f\|_{L^{p}\left(\mathbf{R}^{N}\right)} \equiv\|f\|_{\mathscr{T}_{\alpha}^{p}}<\infty\right\}, \quad \alpha>1 .
\end{aligned}
$$

Here derivatives are interpreted in the weak sense. These spaces and their variants are sometimes called Nikol'skii spaces. If $H_{\alpha}^{p}\left(\mathbf{R}^{N}\right)$ are the usual Sobolev spaces then for any $\varepsilon>0$ it is known that $\Re_{\alpha+\varepsilon}^{p} \subseteq H_{\alpha}^{p} \subseteq \Re_{\alpha}^{p}$. Nikol'skii spaces on domains $U \subseteq \mathbf{R}^{N}$ with, say, $C^{1}$ boundary are simply defined to be the restrictions of functions in $\mathcal{T}_{\alpha}^{p}$. So the above imbedding property persists. See [1] for details on these matters.

Now the analogue of the Main Theorem persists for Nikol'skii spaces. Every step in the proof is the same except that the Minkowski and generalized Minkowski inequalities must be used in place of more elementary estimates used in $\$ \S 2-8$.

R. Goodman [9] has proved versions of some of the above results in the $L^{2}$ norm in a representation-theoretic context.

Ornstein [16] has shown that estimates of the type

do not hold.

$$
\|X Y f\|_{L^{1}} \leqslant C\left\{\left\|X^{2} f\right\|_{L^{1}}+\left\|Y^{2} f\right\|_{L^{1}}\right\}
$$

Ludovich [3] and Mityagin and Semenov [15] have shown that estimates of the type $\|X Y f\|_{\text {sup }} \leqslant C\left\{\left\|X^{2} f\right\|_{\text {sup }}+\left\|Y^{2} f\right\|_{\text {sup }}\right\}$ do not hold. This is why the Main Theorem is formulated in the form $\Lambda_{\alpha}$ at the integer level.

An estimate of the type

$$
\|X Y f\|_{L_{\text {loc }}^{2}} \leqslant C\left\{\left\|X^{2} f\right\|_{L^{2}}+\left\|Y^{2} f\right\|_{L^{2}}\right\}
$$

follows easily from the Plancherel Theorem. By deeper methods, such as the Riesz transforms, a similar estimate holds in $L^{p}, 1<p<\infty$.

We conclude this brief section by completing the proof that an $f$ satisfying the conclusions of the Main Theorem is in $\Gamma_{\alpha}(G)$. Only the case $\alpha \in \mathbf{Z}$ remains to be done. We use Lemma 8.1.

Fix $0 \neq Y \in V_{1}$. Assume that $f$ satisfies the hypotheses and conclusions of the Main Theorem for $\alpha=1$. Let $h \in \mathbf{R}, 0<|h|<1$. Write

$$
\left.\left.\left|\Delta_{h}^{2} f(g \exp Y \cdot)\right|_{0}|\leqslant| \Delta_{h}^{2} f^{0}(g \exp Y \cdot)\right|_{0}|+| \Delta_{h}^{2} f^{1}(g \exp Y \cdot)\right|_{0} \mid
$$


where $f^{0}, f^{1}$ are as in 8.1 and $\varepsilon$ is at our disposal. Then, using the Main Theorem, the last line is not greater than

$$
C \varepsilon|h|^{2 / 3}\left\|f^{0}\right\|_{Y, 2 / 3}+C \varepsilon^{-1}|h|^{4 / 3}\left\|f^{1}\right\|_{Y, 4 / 3} \leqslant C\left(\varepsilon|h|^{2 / 3}+\varepsilon^{-1}|h|^{4 / 3}\right)\left(\sum_{j=1}^{n}\|f\|_{X_{j, 1}}\right) .
$$

Setting $\varepsilon=|h|^{1 / 3}$, yields that

$$
\|f\|_{Y, 1} \leqslant C\left(\sum_{j=1}^{n}\|f\|_{X_{j}, 1}\right)
$$

Since every $h \in G$ is of the form $h=\exp Y \exp W$, some $Y \in V_{1}, W \in g_{2}$, it follows from 9.1 and the triangle inequality that $f \in \Gamma_{1}$.

The result for $\alpha=2,3, \ldots$ now follows by an inductive argument which we omit.

10. An application to interpolation theory. Folland has proved [7] that the spaces $\left\{\Gamma_{\alpha}\right\}_{0<\alpha<\infty}$ form a real scale of interpolation spaces. His techniques can also be used to prove that they form a complex scale of interpolation spaces. The methods of this paper may be used to prove the same theorem. We will first show how a part of this result follows immediately from the Main Theorem:

Proposition 10.1 Let $\left\{Y_{0}, Y_{1}\right\}$ be an interpolation pair. Let $Y_{\theta}^{\mathbf{R}}, Y_{\theta}^{\mathbf{C}}, 0<\theta<1$, be the intermediate spaces computed by the real and complex methods, respectively (see [4]). Let $0<\alpha_{0}<\alpha_{1}<\infty$ and let $T: Y_{0} \cap Y_{1} \rightarrow \Gamma_{\alpha_{0}}(G)$ be a linear operator. Suppose that

$$
\|T f\|_{\Gamma_{\alpha_{j}}} \leqslant C\|f\|_{Y_{j}}, \quad \text { all } f \in Y_{0} \cap Y_{1}, \quad j=0,1 .
$$

Then for $0<\theta<1, f \in Y_{0} \cap Y_{1}$, it holds that

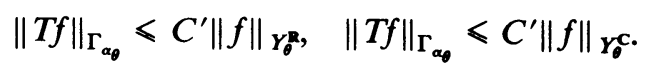

Here $\alpha_{\theta}=(1-\theta) \alpha_{0}+\theta \alpha_{1}$.

Proof. Fix $g \in G$ and $X_{i}$ a basis vector for $V_{1}, 1 \leqslant i \leqslant n$. Consider the operator

$$
T_{g, i}: f \mapsto(T f)\left(g \exp X_{i} \cdot\right) .
$$

Then $T_{g, i}$ is bounded in norm from $Y_{j}$ to $\Lambda_{\alpha_{j}}(\mathbf{R}), j=0,1$. By classical interpolation theorems (see [4]) for $\left\{\Lambda_{\alpha}\right\}_{0<\alpha<\infty}$, it holds that $T_{g, i}$ is bounded from $Y_{\theta}^{\mathbf{C}}$ or $Y_{\theta}^{\mathbf{R}}$ to $\Lambda_{\alpha_{\theta}}(\mathbf{R})$. Since this result holds uniformly over $g \in G, 1<i<n$, it follows from the Main Theorem that $T$ is bounded in norm from $Y_{\theta}^{\mathbf{R}}$ or $Y_{\theta}^{\mathbf{C}}$ to $\Gamma_{\alpha_{\theta}}$.

In fact, by the methods of $\S 8$, we may allow the domain of $T$ to consist of $\Gamma_{\alpha}$ spaces. More precisely, let $\Gamma_{\alpha_{j}}, Y_{j}$ be as in the proposition. Suppose that $T: \Gamma_{\alpha_{1}} \rightarrow$ $Y_{0} \cup Y_{1}$ is a linear operator satisfying

$$
\|T f\|_{Y_{j}}<C\|f\|_{\Gamma_{\alpha_{j}}}, \quad j=0,1, f \in \Gamma_{\alpha_{i}} .
$$

Let $\alpha_{\theta}$ be as in the Proposition and let $f \in \Gamma_{\alpha_{\theta}}$. Assume for simplicity that $V_{1}$ is two dimensional: $V_{1}=\operatorname{span}\left\{X_{1}, X_{2}\right\}$. By the same method as in the proof of 8.1 , there 
is a $C>0$ so that for every $1>\varepsilon>0$ there is a decomposition $f=f^{0}+f^{1}$ with

$$
\begin{aligned}
& \left\|f^{0}\right\|_{X_{j, \alpha_{0}}} \leqslant C \cdot \varepsilon^{\theta} \cdot K(f), \quad j=1,2 ; \\
& \left\|f^{1}\right\|_{X_{j, \alpha_{1}}} \leqslant C \cdot \varepsilon^{\theta-1} \cdot K(f), \quad j=1,2 .
\end{aligned}
$$

As in 8.1,

$$
K(f)=\sum_{j=1}^{2}\|f\|_{X_{j}, \alpha_{0}}+\sum_{\substack{\mathcal{X}=\left\{X_{I_{1}}, \ldots, X_{I_{p}}\right\} \\\left(\operatorname{some} X_{I_{t}} \in g_{2}\right) \\ X_{I} \in g}}\left\|X_{I_{1}} \cdots X_{I_{p}} f\right\|_{X_{I}, \alpha(I) \gamma(\mathcal{X})}
$$

Then $T f=T f^{0}+T f^{1}$. But

$$
\left\|T f^{0}\right\|_{Y_{0}} \leqslant C \cdot\left\|f^{0}\right\|_{\Gamma_{a_{0}}} \leqslant C \sum_{j=1}^{2}\left\|f^{0}\right\|_{X_{j, a_{0}}} \leqslant C \cdot \varepsilon^{\theta} K(f) \leqslant C \cdot \varepsilon^{\theta}\|f\|_{\Gamma_{a_{\theta}}}
$$

by the Main Theorem. Likewise,

$$
\left\|T f^{1}\right\|_{Y_{1}}<C \cdot \varepsilon^{\theta-1}\|f\|_{\Gamma_{a_{\theta}}}
$$

By the definition of real interpolation space,

$$
\|T f\|_{Y_{\theta}^{R}} \leqslant C^{\prime}\|f\|_{\Gamma_{a_{\theta}}} .
$$

So $T$ is a bounded linear operator from $\Gamma_{\alpha_{\theta}}$ to $Y_{\theta}^{\mathbf{R}}, 0<\theta<1$.

11. An application to partial differential equations. We give here a special example to show how the Main Theorem (more precisely its $\mathfrak{T}_{\alpha}^{2}$ variant) already contains information about subelliptic estimates for certain partial differential operators.

For $k=1,2, \ldots$ define $\mathscr{P}_{k}=\sum_{i=1}^{r} X_{i}^{2 k}$. Let $u \in C_{c}^{2 k}(G)$ be real valued and let $f=\mathscr{P}_{k} u$. Assume that the adjoint of $X_{i}$ is $-X_{i}, i=1, \ldots, n$ (this occurs, for instance, on the Heisenberg group with the standard choice of basis for $V_{1}$ ).

Now, letting $d x$ denote Lebesgue (= Haar) measure, we have

$$
\int\left(\mathscr{P}_{k} u\right) \bar{u} d x=\int \sum\left(X_{i}^{2 k} u\right) \bar{u} d x=(-1)^{k} \sum \int X_{i}^{k} u\left(\overline{X_{i}^{k} u}\right) d x
$$

by integration by parts. Fix $1 \leqslant i_{0} \leqslant n$. Then

$$
\int\left|X_{i_{0}}^{k} u\right|^{2} d x=(-1)^{k} \int\left(\mathscr{P}_{k} u\right) \bar{u} d x-\sum_{i \neq i_{0}} \int\left|X_{i}^{k} u\right|^{2} d x
$$

Since the left side is positive and the second term on the right is negative, it follows that

$$
\int\left|X_{i_{0}}^{k} u\right|^{2} d x \leqslant \int\left|\mathscr{P}_{k} u\right||u| d x \leqslant \int|f|^{2} d x+\int|u|^{2} d x
$$

or $\left\|X_{i_{0}}^{k} u\right\|_{L^{2}} \leqslant\|f\|_{L^{2}}+\|u\|_{L^{2}}, 1 \leqslant i_{0} \leqslant n$. By the Nikol'skii space analogue of the Main Theorem, it follows that

$$
\sup _{\substack{\left.X_{I_{1}}, \ldots, X_{I_{p}}\right\} \subseteq g \\ X_{I} \in g \\ g \in G}}\left\|X_{I_{1}} \cdots X_{I_{p}} f\left(g \exp X_{I} \cdot\right)\right\|_{\mathscr{F}_{k(I))(x)}^{2}} \leqslant C\left(\|f\|_{L^{2}}+\|u\|_{L^{2}}\right) .
$$


In particular, by the comparson of Nikol'skii and Sobolev spaces,

$$
\|u\|_{\left(H_{k / m-\varepsilon}^{2}\right)} \leqslant C_{\varepsilon}\left(\|f\|_{L^{2}}+\|u\|_{L^{2}}\right), \text { any } \varepsilon>0 .
$$

A more complete theory of subelliptic estimates may be obtained by exploiting the techniques of $\S \S 2-8$. However this topic exceeds the scope of the present paper.

It should be noted that (11.1) still holds even if the $X_{i}$ are not selfadjoint. For then the integration by parts gives rise to lower order terms which are easily absorbed.

Operators of the type $\Sigma X_{i}^{2}$ have been studied in [11], [8], [6], [17].

12. An application to function theory. With $G$ as usual, $0<k \in \mathbf{Z}$, let

$$
e^{k}(G)=\left\{\gamma:(0,1) \rightarrow G|| \gamma^{(1)}(t)|\leqslant 1, \ldots,| \gamma^{(k)}(t) \mid<1 \text {, all } t \in(0,1)\right\} .
$$

Here $\gamma^{(j)}(t)$ denotes the $j$ th derivative on $(0,1)$. For $1 \leqslant i \leqslant m$, set

$$
\mathcal{C}_{i}^{k}(G)=\left\{\gamma \in \mathcal{C}^{k}(G): \dot{\gamma}(t) \in \mathrm{g}_{i}\right\} \text {. }
$$

Proposition 12.1. Let $0<\alpha<\infty$ and $\alpha<k \in \mathbf{Z}$. Then $f \in \Gamma_{\alpha}(G)$ if and only if there is a $C>0$ so that for all $\gamma \in \mathcal{C}_{i}^{k}, 1 \leqslant i \leqslant m$, one has that $\|f \circ \gamma\|_{\Lambda_{\alpha / i}(0,1)}<C$.

PROOF. In case $\|f \circ \gamma\|_{\Lambda_{\alpha / i}(0,1)} \leqslant C$, all $\gamma \in \mathcal{C}_{i}^{k}, 1<i<m$, the hypotheses of the Main Theorem are satisfied (since $t \mapsto g \exp X_{l} t$ is in $C_{1}^{k}, 1<l<n$ ) so $f \in \Gamma_{\alpha}$.

Conversely, fix $\gamma \in \mathcal{C}_{i}^{k}$. Suppose $\alpha \notin \mathbf{Z}$. If $f \in \Gamma_{\alpha}$ then $f \circ \gamma \in \Lambda_{\alpha / i}$ by the definition of $\Gamma_{\alpha}$ and the Chain Rule. The result for $\alpha \in \mathbf{Z}$ now follows by interpolation.

This proposition is weaker than the Main Theorem. However, it provides a natural and intrinsic characterization of the $\Gamma_{\alpha}$. Results of this kind were explored in [8] in the case of the Heisenberg group.

\section{REFERENCES}

1. Robert Adams, Sobolev spaces, Academic Press, New York, 1975.

2. J. Bergh and B. Löfström, Interpolation spaces: An introduction, Springer, Berlin, 1976.

3. O. Besov, V. Ilin and S. Nikol'skii, Integral representations of functions and imbedding theorems, Vols. I, II, Wiley, New York, 1978.

4. Paul Butzer and Hubert Berens, Semi-groups of operators and approximation, Springer, New York, 1967.

5. A. P. Calderon, Intermediate spaces and interpolation, the complex method, Studia Math. 24 (1964), 113-190.

6. G. B. Folland, Subelliptic estimates and function spaces on nilpotent lie groups, Ark. Math. 13 (1975), 161-207.

7. Lipschitz classes and Poisson integrals on stratified groups, Studia Math. 66 (1979), 37-55.

8. G. B. Folland and E. M. Stein, Estimates for the $\bar{\partial}_{b}$ complex and analysis on the Heisenberg group, Comm. Pure Appl. Math. 27 (1974), 429-522.

9. R. Goodman, Some regularity theorems for operators in an enveloping algebra, J. Differential Equations 10 (1971), 448-470.

10. G. Hochschild, The structure of Lie groups, Holden-Day, San Francisco, Calif., 1965.

11. L. Hörmander, Hypoelliptic second order differential equations, Acta Math. 119 (1967), 147-171.

12. S. G. Krantz, Structure and interpolation theorems for certain Lipschitz classes and estimates for the $\bar{\partial}$ equation, Duke Math. J. 43 (1976), 417-439.

13. , Intrinsic Lipschitz classes on manifolds with applications to complex function theory and estimates for the $\bar{\partial}$ and $\bar{\partial}_{b}$ equations, Manuscripta Math. 24 (1978), 351-378.

14. Geometric Lipschitz spaces and applications to complex function theory and nilpotent groups, J. Funct. Anal. 34 (1979), 456-471. 
15. B. Mityagin and E. Semenov, The space $C^{k}$ is not an interpolation space between $C$ and $C^{n}$, $0<k<n$, Soviet Math. Dokl. 17 (1976), 778-782.

16. D. Ornstein, A non-inequality for differential operators in the $L^{1}$ norm, Arch. Rational Mech. Anal. 11 (1962), 40-49.

17. L. P. Rothschild and E. M. Stein, Hypoelliptic differential operators and nilpotent groups, Acta Math. 137 (1977), 247-320.

18. E. M. Stein, Singular integrals and differentiability properties of functions, Princeton Univ. Press, Princeton, N. J., 1970.

19. A. Zygmund, Trigonometric series, Cambridge Univ. Press, Cambridge, 1968.

Department of Mathematics, Pennsylvania State University, University Park, Pennsylvania 16802 